\title{
FUZZY MULTIPLE OBJECTIVE FRACTIONAL OPTIMIZATION IN ROUGH APPROXIMATION AND ITS APTNESS TO THE FIXED-CHARGE TRANSPORTATION PROBLEM
}

\author{
Sudipta Midya ${ }^{1}$, Sankar Kumar Roy ${ }^{1, *}$ And Gerhard Wilhelm Weber ${ }^{2}$
}

\begin{abstract}
This article presents a multiple objective fractional fixed-charge transportation problem (MFFTP) in a rough decision-making framework. A transformation procedure is modified to convert non-linear multi-objective transportation problem to its linear version. The parameters of the designed model are considered to be fuzzy. We employ separate kinds of fuzzy scale, i.e., possibility, credibility and necessity measures, to deal with the fuzzy parameters. Using the fuzzy chance-constrained rough approximation (FCRA) technique, we extract the more preferable optimal solution from our suggested MFFTP. The initial result is compared with that of the robust ranking (RR) technique. We also use the theory of rough sets for expanding as well as dividing the feasible domain of the MFFTP to accommodate more information by considering two approximations. Employing these approximations, we introduce two variants, namely, the lower approximation (LA) and the upper approximation (UA), of the suggested MFFTP. Finally, by using these models, we provide the optimal solutions for our proposed problem. We also associate our MFFTP with a real-world example to showcase its applicability as well as performance. Our core concept of this article is that it tackles an MFFTP using two separate kinds of uncertainty and expands its feasible domain for optimal solutions. Optimal solutions of the designed model (obtained from FCRA technique) belong to two separate regions, namely, "surely region" and "possible region". The optimal solution which belongs to the "surely region" is better (as these are minimum values) than the one in the "possible region" and other cases. An interpretation of our approach along with offers about the intended future research work are provided at last.
\end{abstract}

Mathematics Subject Classification. 90C32, 90C29, 90C08, 90C70.

Received December 27, 2020. Accepted May 3, 2021.

\section{INTRODUCTION}

Fractional programming is a special type of non-linear platform in which the quotients of functions are either maximized or minimized. The study of fractional programming started long before with Charnes and Cooper [6] being the first. Fractional programming for the single-objective optimization problem was investigated

Keywords. Fractional programming, fixed-charge transportation problem, rough programming, fuzzy programming, robust ranking technique, fuzzy chance-constrained rough technique.

1 Department of Applied Mathematics with Oceanology and Computer Programming, Vidyasagar University, Midnapore 721102, West Bengal, India.

2 Faculty of Engineering Management Poznan University of Technology, ul. Jacka Rychlewskiego 2, 60-965 Poznan, Poland.

3 IAM (UME), Middle East Technical University, 06800 Ankara, Turkey.

*Corresponding author: sankroy2006@gmail.com

(C) The authors. Published by EDP Sciences, ROADEF, SMAI 2021 
extensively and applied to real-life problems, for example, economical planning in corporate sector, transportation scheduling, university planning, and health care and hospital management, etc. In these areas, fractional programming is frequently encountered through the decision to optimize ratios such as profit/invest, damaged rate of goods/time, placement/student, nurse/patient and others.

In the traditional transportation problem (TP), single product is manufactured at different plants (supply points) and transported to a number of different warehouses (demand points). The objective is to satisfy the demand of the goods moved from each supply center to every demand center in which the cost of transportation takes minimum value. Besides the shipping cost, in many real-life applications, a TP is usually connected to a fixed penalty which is incurred at each origin. That fixed penalty may be due to setup expenses, permit charge, toll fee and others. This problem is called the fixed-charge transportation problem (FCTP).

Since the TP is a special case within the class of linear programming problems, it is expressed as a linear fractional programming problem (FPP) which has a network designed containing of definite numbers of vertices and curves. This kind of TP is called a fractional transportation problem (FTP). A linear FTP seeks to optimize an objective function of fractional form, with linear functions appearing in its numerator and denominator, subject to a set of linear constraints.

Fractional fixed-charge transportation problem (FFTP) is the enhanced structure of FTP which is formulated by including a fixed-charge into the transportation system. Generally, many real-world situations also arise for which a single-objective optimization setup is not adequate. Based on this fact multiple objective functions are considered into the FFTP and it is further modified to multiple objective fractional fixed-charge TP. Therefore, multiple objective case of the proposed model puts it ahead of its single-objective counterpart. In the formulated multiple objective fractional fixed-charge transportation problem (MFFTP), multiple objective functions are of conflicting type and of ratio form. So, sometimes our problem is called non-linear multiple objective FCTP. Generally, this type of FCTP is hard to solve. Due to this reason, we use suitable transformation and modify it to convert the model into its linear form.

In real-life delivery system, uncertainty exists almost every cases. To tackle such types of uncertainty in practical situations, researchers have adopted it in various ways such as stochastic, interval, fuzzy, rough, etc. environments. Fuzzy set was launched by Zadeh [55] as a powerful mathematical aid for representing inexactness, inconsistency and imprecise data in real-world situations whereas rough set theory, on the another way, was originated by Pawlak [36]. It is generally demonstrated to be a strong mathematical equipment to represent vagueness in the optimization problems.

To tackle uncertain parameters in a TP, one can consider stochastic framework. But, stochastic distribution is required either a priori predictable periodicity or posteriori frequency distribution. However, many situations appear in practical transportation system where we have no information about predictable periodicity/frequency distribution of the parameters. In contrast, in fuzzy set theory, that information is not needed such type of predictable periodicity/frequency distribution. Moreover, fuzzy set theory is expressed everything by degree of membership function. Due to these reasons, the parameters of the formulated model are taken as fuzzy number instead of using a stochastic environment.

As in fuzzy set the inexact information is described by membership function so it is easy to present uncertainty in real-life distribution system by fuzzy numbers. Behind this reason in our suggested model the variables are regarded as triangular fuzzy numbers, so the MFFTP is again defined as fuzzy MFFTP. We also incorporate rough set theory to divide the feasible region of the fuzzy MFFTP. Basically, rough set approximation is produced the two correspondence models (i.e., linear lower approximation (LA) and upper approximation (UA) of the MFFTP) which are connected with the possibility and necessity of chance constraints. These two models provide two sets of optimal solutions in the sense of "surely" and "possible" situations respectively in the practical delivery system. Moreover, the chance operator is used to reduce it into a deterministic form.

From publications view, it is noticed that until now no one did consider a fuzzy MFFTP through rough approximation which motivated us to design an MFFTP model. Main attention of our efforts to design this article is depicted as below. 
- MFFTP model is developed by introducing two kinds of uncertainty, (i.e., one is fuzziness and another is roughness);

- A modification is done to convert MFFTP to $\operatorname{MFCTP}$ (i.e., linearization process);

- Transform the fuzzy non-linear multiple objective fixed-charge transportation problem (MFCTP) into the fuzzy linear MFCTP;

- $\mathrm{RR}$ and fuzzy chance-constrained rough approximation (FCRA) techniques are employed to formulate an equivalent crisp version of the fuzzy MFFTP;

- The feasible region of the proposed MFFTP is extended by formulating two approximation models, namely, the linear LA and UA of the MFFTP (i.e., LLA-MFCTP and LUA-MFCTP), using rough set approximation, that creates the police-making process more acceptable;

- Comparison among the optimal solutions extracted from RR, FCRA techniques and the existing approach.

Remainder of the article is structured as follows. In Section 1.1, a literature survey is discussed. Ground behind this study is described in Section 1.2. In Section 2, fundamental concept of rough set, fuzzy number and related definitions, theorems and lemma are depicted. In Section 3, the fuzzy MFFTP and the linear fuzzy MFCTP are introduced. Section 4 proposes a deterministic version of the fuzzy MFFTP. Deficiency of the existing methods are noticed in Section 5. The solution procedure of our proposed MFFTP model and bi-objective FFTP are presented in Section 6. An application example to the fuzzy MFFTP is given in Section 7.1, and we discuss the results of all equivalent crisp versions of the proposed MFFTP in Section 7.2. Managerial benefits of the proposed study are presented in Section 8. Finally, we conclude and give some forward-looking remarks in Section 9 .

\subsection{Literature survey}

The FPP with fixed-charge was initiated by Almogy and Levin [1] whereas FCTP was originated by Hirsch and Dantzig [18]. After their work, a good number of research papers has been published on FPP/FTP. A few of them are described as below.

Schaible [42] presented fractional programming and its duality. Chakraborty and Gupta [7] solved multiple objective linear FPP by fuzzy mathematical platform. Mishra [32] described bi-level FPP and solved it by weighting approach. Toksari [46] presented Taylor series method for fuzzy multiple objective linear FPP. Mishra et al. [33] used fuzzy multiple FPP for land and agricultural production planning. Jiao and Liu [19] introduced a new linearization technique for minmax linear FPP. Anukokila et al. [2] solved fuzzy multi-objective fractional transportation problem. Mahmoodirad et al. [25] designed a linear fractional TP under uncertain environment. In recent years, many researchers have studied on FPP/FTP by considering the parameters of FTP as either a crisp or an interval or a fuzzy value, such as Chang [8], Bhati and Singh [5], Das et al. [9], Ebrahimnejad et al. [11], Sivri et al. [43] and Arya et al. [3].

In the last few decades, many researchers have shown significant interest in uncertain environment to tackle uncertainty in FTP and FCTP. Furthermore, many studies have been focused on multi-objective transportation problem. For example, Midya and Roy ([28], [29]) solved single-sink FCTP under stochastic environment, and analyzed FCTP under interval and rough interval environment. Upmanyu and Saxena [47] presented an imprecise fractional objective functions and solved a multiple objective problem with fixed-charge. Maity and Roy [26] solved a multiple objective TP by considering multi-choice demand and cost function is of non-linear type. Xie and Jia [51] solved the FCTP by using a genetic algorithm. Roy et al. [37] presented a conic scalarization technique to solve multiple objective TP under multi-choice ground. Sagratella et al. [41] designed a noncooperative FCTP by introducing a game-theoretic extension. Tao and Xu [45] developed rough programming to tackle multiple objective solid TP, whereby they discovered that the feasible domain was not fixed but more workable due to impreciseness of parameters. Vasant et al. [49] introduced advance optimization techniques and applications in economics and engineering. Roy and Midya [39] designed an intuitionistic fuzzy multiple objective fixed-charge solid TP with product blending. Anukokila et al. [2] solved fuzzy multi-objective fractional 
TABLE 1. List of notable related publications of FTP.

\begin{tabular}{llllll}
\hline \hline Authors & $\begin{array}{l}\text { Characteristic } \\
\text { of problem }\end{array}$ & $\begin{array}{l}\text { Extra } \\
\text { function }\end{array}$ & Framework & $\begin{array}{l}\text { Type of } \\
\text { Optimization }\end{array}$ & $\begin{array}{l}\text { No. of } \\
\text { goals }\end{array}$ \\
\hline Arya et al. [3] & FPP & - & Fuzzy & Maximization & Multiple \\
Bhati and Singh [5] & FPP & - & Crisp & Maximization & Multi \\
Chakraborty and Gupta [7] & FPP & - & Crisp & Maximization & Multi \\
Chang [8] & FPP & - & Fuzzy & Maximization & Multi \\
Das et al. $[9]$ & FPP & - & Fuzzy & Maximization & Single \\
Ebrahimnejad et al. [11] & FPP & - & Fuzzy & Maximization & Single \\
Jiao and Liu [19] & FPP & - & Crisp & Maximization & Single \\
Mahmoodirad et al. [25] & FTP & - & Uncertain & Minimization & Single \\
Mishra [32] & FPP & - & Crisp & Maximization & Single \\
Mishra et al. [33] & FPP & - & Fuzzy & Maximization & Multi \\
Sivri et al. [43] & FTP & - & Crisp & Minimization & Single \\
Toksari [46] & FPP & - & Fuzzy & Maximization & Multi \\
Upmanyu and Saxena $[47]$ & FPP & Fixed-charge & Fuzzy & Minimization & Multi \\
Suggested model & FTP & Fixed cost & Fuzzy and rough & Minimization & Multiple \\
\hline
\end{tabular}

transportation problem. Ghosh et al. [12] formulated a multi-objective fixed-charge solid TP under intuitionistic fuzzy environment.

To tackle uncertain parameters in practical distribution systems, researchers have studied in different ways. A few of them are as follows. Paksoy et al. [34] investigated an application of fuzzy optimization to a supply chain network design. Goli and Malmir [16] presented a covering tour approach for disaster relief locating and routing with fuzzy demand. Goli et al. [17] designed a fuzzy integrated cell formation and production scheduling by considering human factors and automated guided vehicles. Tirkolaee et al. [48] used a Pareto-based algorithms to solve a multi-objective optimization for the reliable pollution-routing problem with cross-dock selection. Goli et al. [14] solved a fuzzy mathematical model under multi-objective ground for a financially constrained closed-loop supply chain. Ghosh and Roy [13] solved a multi-objective product blending FCTP with truck load constraints through transfer station under a fuzzy-rough framework.

In reality some situations arise where two types of uncertainty occur simultaneously. Based on this fact researchers have studied two-fold uncertainty environment. For instance, Roy et al. ([38], [40]) designed an MFCTP under random rough environment and multiple objective solid TP with fixed-charge in fuzzy rough environment. $\mathrm{Xu}$ and Zhao [53] studied a multiple objective decision making problem with fuzzy rough variables and solved the inventory problems. Moreover, Midya \& Roy [30] solved an MFCTP using rough programming. Midya et al. [31] designed a multi-stage multi-objective fixed-charge solid TP under intuitionistic fuzzy framework through a green supply chain. Veeramani and Sumathi [50] solved a fuzzy linear fractional programming problem. Maity et al. [27] solved a dual-hesitant fuzzy TP under restrictions by introducing a technique. Kaur et al. [20] formulated a restricted flow capacitated two-stage time minimization transportation problem. Goli et al. [15] presented a hybrid artificial intelligence and robust optimization for a multi-objective product portfolio problem. Paul et al. [35] studied effect of price-sensitive demand and default risk on optimal credit period and cycle time for a deteriorating inventory model. A prompt list of some recent articles related to FTP is displayed in Table 1.

\subsection{Motivation for this study}

Fuzzy set and rough set theories are two separate kinds of mathematical aids to tackle ambiguous and vague information in reality. Fuzzy set theory presents the inexact information by membership function which belongs to $[0,1]$ whereas rough set theory measures vague information by the approximation of two ordinary sets namely, LA and UP sets. The LA set captures the data in surely belonging sense and the UP set codes the data in possible 
belonging sense. In the proposed MFFTP model, the parameters are taken as fuzzy numbers of triangular type to describe the imprecise parameter in a membership grade and rough set theory is used to expand the feasible domain of optimal solution by LA and UA concepts.

In real-world situations, several factors are defined to the objective functions and constraints to formulate an MFFTP model. Usually, the variables in the objective functions and constraints are inexact or vague in nature due to fluctuations in economic and financial variables, insufficient information about input data, weather condition and other inconsiderable factors. For these cases, the values of the parameters are taken based on decision makers (DMs) or experts opinions which are generally not an exact number but it is an interval value or a linguistic term. These indeterministic parameters of an MFFTP can be expressed by fuzzy numbers. In this stand point, the parameters, i.e., variable and fixed transportation costs, amount of profit, damaged rate of goods, time of transportation, size of sources, and demands are treated as triangular fuzzy numbers.

It is more preferable for an organization/company if the feasible space of an optimal strategy (i.e., an optimal solution) can be expanded due to highly market competition in global economy. The fundamental concept of a rough set is characterized by a pair of exact ideas such as interval, set etc. which is referred to as LA and UA of the set. To extend as well as the partition in the feasible region of a fuzzy MFFTP, rough approximation (i.e., LA and UA) technique is adopted to improve our optimization algorithm which makes decision-making process more flexible. Thus, proposed MFFTP has a realistic background to handle the uncertain information in real-life systems.

Some practical circumstances appear where ratio optimization is required in police-making systems. For instance, when the grape (one kind of fruit) is transported, the damaged rate of grapes is happened with respect to time. The goals of an organization's DM are to minimize: total cost concerning to total investment, damaged rate of commodities with respect to time and others. In this standpoint, fractional goal functions are adopted in FCTP. So, behind considering ratio optimization in our suggested model has a practical setting. Thus, by accommodating the fuzzy and rough set theories and ratio programming, we are able to reflect in the constructed MFFTP as many attributes of the practical problem as possible, which is indeed the central motivation of this paper.

\section{Preparatories}

Herein, we discuss the primary concept of rough set and fuzzy set. Moreover, helpful definitions, theorems and lemma related to fuzzy and rough variables are presented.

\subsection{Rough set and its approximation}

Consider a set of elements $U$ which is called the "universe". The deficiency of knowledge about the objects of $U$ is represented by an indiscernibility relation, $R \subseteq U \times U$. Because of simplicity, an equivalence relation $R$ is considered. Assuming $Y$ is a subset of $U$. Our intension is to identify the set $Y$ to relate to $R$. The primary concepts of rough sets are described below:

Definition 2.1. Set $Y$ is said to be precise (i.e., crisp) to relate to $R$, if the border area of $Y$ is empty. Set $Y$ is said to be imprecise (i.e., rough) to relate to $R$, if the frontier area of $Y$ is nonempty.

$R(y)$ is indicated an equivalence class of $R$ which is defined by any of its elements $y$. Our insufficiency knowledge about universe in surely sense is expressed by an indiscernibility relation. Equivalence classes of indiscernibility relation, said to be granules of knowledge, are generated by $R$ and they represent a basic part of knowledge.

Definition 2.2. [52] Lower approximation of $Y$ to relate to $R$ is symbolically represented by $\underline{R}(Y)$ and is characterized as below:

$$
\underline{R}(Y):=\bigcup_{y \in U}\{R(y): R(y) \subseteq Y\}
$$


Upper approximation of $Y$ to relate to $R$ is indicated by $\bar{R}(Y)$ and is described as stated below:

$$
\bar{R}(Y):=\bigcup_{y \in U}\{R(y): R(y) \cap Y \neq \phi\} .
$$

Boundary region of $Y$ to relate to $R$ is symbolized by $B N_{R}(Y)$ and is depicted as below:

$$
B N_{R}(Y):=\bar{R}(Y)-\underline{R}(Y) .
$$

\subsection{Fuzzy numbers}

Definition 2.3. (Fuzzy Number) [57]. Let $\tilde{A}$ be a fuzzy set described on $\mathbb{R}$ (real numbers set), is called to be a fuzzy number if its membership function $\mu_{\tilde{A}}: \mathbb{R} \rightarrow[0,1]$ satisfies the following criteria:

- $\mu_{\tilde{A}}$ is a convex, i.e., $\mu_{\tilde{A}}\left\{\lambda x_{1}+(1-\lambda) x_{2}\right\} \geq \min \left\{\mu_{\tilde{A}}\left(x_{1}\right), \mu_{\tilde{A}}\left(x_{2}\right)\right\}$ for all $x_{1}, x_{2} \in \mathbb{R}, 0 \leq \lambda \leq 1$;

- $\mu_{\tilde{A}}$ is a normal, i.e., there is an $x \in \mathbb{R}$ such a way $\mu_{\tilde{A}}(x)=1$;

- $\mu_{\tilde{A}}$ is a piecewise continuous.

Definition 2.4. (Triangular Fuzzy Number) [57]. A triangular fuzzy number is a fuzzy number which is described with three points as $\tilde{A}=\left(a_{1}, a_{2}, a_{3}\right)$, where $a_{1}, a_{2}, a_{3} \in \mathbb{R}$. This triplet is performed a membership function which is symbolized as $\mu_{\tilde{A}}(x)$. It satisfies the following conditions:

- $\mu_{\tilde{A}}(x)$ is an increasing function in $a_{1}$ to $a_{2}$;

- $\mu_{\tilde{A}}(x)$ is a decreasing function in $a_{2}$ to $a_{3}$;

- $a_{1}<a_{2}<a_{3}$.

So, the membership function is depicted as follows:

$$
\mu_{\tilde{A}}(x)= \begin{cases}0, & \text { if } x<a_{1} \\ \frac{x-a_{1}}{a_{2}-a_{1}}, & \text { if } a_{1} \leq x \leq a_{2}, \\ \frac{a_{3}-x}{a_{3}-a_{2}}, & \text { if } a_{2} \leq x \leq a_{3}, \\ 0, & \text { if } x>a_{3}\end{cases}
$$

\subsubsection{Arithmetic operation between fuzzy numbers}

Assuming two triangular fuzzy numbers are $\tilde{A}=\left(a_{1}, a_{2}, a_{3}\right)$ and $\tilde{B}=\left(b_{1}, b_{2}, b_{3}\right)$. Addition and subtraction of the triangular fuzzy numbers are presented as follows:

$$
\begin{gathered}
\tilde{A}+\tilde{B}=\left(a_{1}+b_{1}, a_{2}+b_{2}, a_{3}+b_{3}\right), \\
\tilde{A}-\tilde{B}=\left(a_{1}-b_{3}, a_{2}-b_{2}, a_{3}-b_{1}\right) .
\end{gathered}
$$

Definition 2.5. ( $\alpha$-cut) [57]. The $\alpha$-level set ( $\alpha$-cut) of a fuzzy number $\tilde{A}$ is a crisp set is $\operatorname{denoted}$ by $[\tilde{A}]^{\alpha}$ and it is represented as follows:

$$
[\tilde{A}]^{\alpha}=\left\{x: \mu_{\tilde{A}}(x) \geq \alpha \forall \alpha \in(0,1)\right\} .
$$

Let $\tilde{M}$ be a fuzzy number, then $[\tilde{M}]^{\gamma}$ is denoted as a closed interval of $\mathbb{R}$ for all $\gamma \in(0,1)$. Here, we treat an another form of $[\tilde{M}]^{\gamma}$ by

$$
[\tilde{M}]^{\gamma}=\left[m_{1}(\gamma), m_{2}(\gamma)\right] \subseteq \mathbb{R}
$$

where lower and upper limits of $\tilde{M}$ are $m_{1}(\gamma)$ and $m_{2}(\gamma)$.

Remark 2.6. The $\gamma$-cut of a triangular fuzzy number $\tilde{A}=(a, \alpha, \beta)$ (where $\alpha$ and $\beta$ are the left and right spreads of $\tilde{A})$, is an interval denoted by $[\tilde{A}]^{\gamma}$, and it is represented as follows:

$$
[\tilde{A}]^{\gamma}=[a-(1-\gamma) \alpha, a+(1-\gamma) \beta] \quad(\gamma \in[0,1]) .
$$


Definition 2.7. [10]. Assuming $\varphi$ is a function defined on $\mathbb{R}$ (real numbers set) to $[0,1]$. Then, $\varphi$ is called the reference function of a triangular fuzzy variable, if $\varphi$ satisfies the criteria stated as below:

- $\varphi(x)=\varphi(-x) \quad \forall x \in \mathbb{R}$;

- $\varphi(0)=1$

- $\varphi$ is decreasing on $[0, \infty)$.

Definition 2.8. [10]. Let $\tilde{a}_{1}, \tilde{a}_{2}, \ldots, \tilde{a}_{m}$ be fuzzy variables, and $f: \mathbb{R}^{n} \rightarrow \mathbb{R}$ be a function which is continuous. Then, possibility of the fuzzy event specified by $f\left(\tilde{a}_{1}, \tilde{a}_{2}, \ldots, \tilde{a}_{m}\right) \leq 0$ is defined by

$$
\operatorname{Pos}\left\{f\left(\tilde{a}_{1}, \tilde{a}_{2}, \ldots, \tilde{a}_{m}\right) \leq 0\right\}=\sup _{x_{1}, x_{2} \ldots, x_{m}}\left[\min _{1 \leq i \leq m} \mu_{\tilde{a}_{i}}\left(x_{i}\right): f\left(\tilde{x}_{1}, \tilde{x}_{2}, \ldots, \tilde{x}_{m}\right) \leq 0\right] .
$$

Definition 2.9. [10]. Let $(\Theta, P(\Theta)$, Pos) be a possibility space, and $\mathrm{A}$ indicate a set in $P(\Theta)$. Then, necessity measure of $\mathrm{A}$ is presented by Nec of A and is defined as follows:

$$
\operatorname{Nec}\{A\}=1-\operatorname{Pos}\left\{A^{c}\right\}
$$

where $A^{c}$ is the complement of $\mathrm{A}$.

Thus, $\operatorname{Pos}\{A\}+\operatorname{Nec}\left\{A^{c}\right\}=1$ for an arbitrary $A \in P(\Theta)$.

Definition 2.10. [24]. Let $(\Theta, P(\Theta)$, Pos) be a possibility space, and A represent a set in $P(\Theta)$. Then, credibility measure of $\mathrm{A}$ is presented as below:

$$
\operatorname{Cr}\{A\}=\frac{1}{2}[\operatorname{Pos}\{A\}+\operatorname{Nec}\{A\}] .
$$

Thus, $\operatorname{Cr}\{A\}+\operatorname{Cr}\left\{A^{c}\right\}=1$ for an arbitrary $A \in P(\Theta)$.

Lemma 2.11. [10]. Let $\zeta_{1}$ and $\zeta_{2}$ be two fuzzy variables. Then, we have

$\operatorname{Pos}\left\{\zeta_{1} \geq \zeta_{2}\right\}=\sup \left\{\min \left(\mu_{\zeta_{1}}(a), \mu_{\zeta_{2}}(b)\right): a, b \in \mathbb{R}, a \geq b\right\}$ and

$\operatorname{Pos}\left\{\zeta_{1}>\zeta_{2}\right\}=\sup \left\{\min \left(\mu_{\zeta_{1}}(a), \mu_{\zeta_{2}}(b)\right): a, b \in \mathbb{R}, a>b\right\}$.

Now it becomes more of a definition, if the decision maker prefers a pessimistic decision in order to avoid risk, for those case the possibility measure is replaced by necessity measure:

$N e c\left\{\zeta_{1} \geq \zeta_{2}\right\}=\inf \left\{\max \left(\left(1-\mu_{\zeta_{1}}(a)\right), \mu_{\zeta_{2}}(b)\right): a, b \in \mathbb{R}, a \geq b\right\}$ and

$N e c\left\{\zeta_{1}>\zeta_{2}\right\}=\inf \left\{\max \left(\left(1-\mu_{\zeta_{1}}(a)\right), \mu_{\zeta_{2}}(b)\right): a, b \in \mathbb{R}, a>b\right\}$.

Proof. Please refer to [10].

Theorem 2.12. [22]. Let $(\Theta, P(\Theta), P o s)$ be a possibility space, and $A$ be a set in $P(\Theta)$. Then, $\operatorname{Pos}\{A\} \geq$ $\operatorname{Cr}\{A\} \geq \operatorname{Nec}\{A\}$.

Proof. First we prove that $\operatorname{Pos}\{A\} \geq \operatorname{Nec}\{A\}$. If $\operatorname{Pos}\{A\}=1$, then it is evident that $\operatorname{Pos}\{A\} \geq \operatorname{Nec}\{A\}$. Besides, we have $\operatorname{Pos}\left\{A^{c}\right\}=1$, which implies that $\operatorname{Nec}\{A\}=1-\operatorname{Pos}\left\{A^{c}\right\}=0$. Thus, $\operatorname{Pos}\{A\} \geq \operatorname{Nec}\{A\}$ holds. Again, it is noticed from Definition 2.10 that the credibility scale takes the middle value of possibility and necessity scales. Hence, $\operatorname{Pos}\{A\} \geq \operatorname{Cr}\{A\} \geq \operatorname{Nec}\{A\}$.

\section{The MATHEMATiCAL MODEL}

Here, we start by describing the basic model and introducing various extensions to formulate our suggested MFFTP. Finally, we develop a fuzzy MFFTP model and convert it to fuzzy linear MFCTP model. Throughout the section, $m, n$ and $t$ denote the number of origins (i.e., source points), destinations (i.e., demand points) and the number of objective functions, respectively. Some essential definitions and properties related to rough sets and fuzzy numbers that are used in this text are provided in Section 2. 


\subsection{The MFCTP}

The FCTP is the extended version of an ancient TP. FCTP is associated with two types of cost, namely, a variable shipping cost (i.e., a direct cost) and a fixed-charge, for moving the product from ith supply center to $j$ th demand center in such a way that the total transportation cost is minimal. In a real-world problem, usually multiple goals are more acceptable than single goal. To adopt these goals, multiple objective functions are considered concurrently in the FCTP. For instance, multiple objective functions i.e., total (shipping and fixed) cost, time of transportation, damaged rate of goods, etc. are minimized. Due to this reason, generally, an MFCTP, involves multiple, conflicting, and incommensurable objective functions subject to a common set of constraints. Hence, the mathematical models of MFCTP (i.e., Model 1) are stated as below.

\section{Model 1}

$$
\begin{aligned}
\operatorname{minimize} & \left(z_{K}: K=1,2, \ldots, t\right) \\
& \text { or }\left[\sum_{i=1}^{m} \sum_{j=1}^{n}\left[z_{1}\left(x_{i j}, \eta\left(x_{i j}\right)\right)\right]\right. \\
& \left.\sum_{i=1}^{m} \sum_{j=1}^{n}\left[z_{K}\left(x_{i j}\right): z=2,3, \ldots, t\right]\right] \\
\text { subject to } & \sum_{j=1}^{n} x_{i j} \leq a_{i} \quad(i=1,2, \ldots, m), \\
& \sum_{i=1}^{m} x_{i j} \geq b_{j} \quad(j=1,2, \ldots, n), \\
& x_{i j} \geq 0, \quad \forall i, j,
\end{aligned}
$$

with $z_{K}$ being the $K$ th objective function $(K=1,2, \ldots, t), x_{i j}$ is the quantity of product moved from source $i$ to destination $j, \eta\left(x_{i j}\right)$ the binary variable taking a unit value if source $i$ is used and 0 otherwise, and $a_{i}$ and $b_{j}$ are the capacity of supply center $i$ and demand center $j$, respectively. The first objective function $z_{1}$ is called the "cost objective function" and defined as follows:

$$
z_{1}\left(x_{i j}, \eta\left(x_{i j}\right)\right)=\left(c_{i j} x_{i j}+f_{i j} \eta\left(x_{i j}\right)\right),
$$

where

$$
\eta\left(x_{i j}\right)=\left\{\begin{array}{l}
0 \text { if } x_{i j}=0 \\
1 \text { if } x_{i j}>0
\end{array}\right.
$$

Here, we denote by $c_{i j}$ and $f_{i j}$ the variable unit cost of transportation and fixed-charge to move the product from supply center $i$ to demand center $j$, respectively. Feasibility criterion of Model 1 is stated as:

$$
\sum_{i=1}^{m} a_{i} \geq \sum_{j=1}^{n} b_{j} .
$$

The first constraints of Model 1 require that the total quantity of item moved from the $i$ th supply center does not exceed its capacity $a_{i}$, whereas the second constraints require the total quantity of product moved from all the sources fulfills the order of product at $j$ th demand center.

\subsection{Multi-objective fractional programming problem}

In a multiple objective FPP (MFPP), all the objective functions are of rational forms, and again, the objective functions are optimized against a set of common constraints. The strength of fractional programming, 
in general, and the MFPP, in particular, is that the value to be optimized is the ratio of different objectives (e.g., minimizing costs and maximizing investment) which together describe the efficiency of the system. The general model of MFPP (i.e., Model 2) is given as follows:

\section{Model 2}

$$
\begin{aligned}
& \text { minimize }\left(Z_{K}(x): K=1,2, \ldots, t\right) \\
& \text { or }\left(\frac{f_{K}(x)}{g_{K}(x)}: K=1,2, \ldots, t\right), \\
& \text { or }\left(\frac{f_{1}(x)}{g_{1}(x)}, \frac{f_{2}(x)}{g_{2}(x)}, \ldots, \frac{f_{t}(x)}{g_{t}(x)}\right) \\
& \text { subject to } \quad v_{l_{1}}(x)\left(\begin{array}{l}
\leq \\
= \\
\geq
\end{array}\right) 0\left(l_{1}=1,2, \ldots, Q\right), \\
& \\
& x \geq 0,
\end{aligned}
$$

where $x$ is the decision parameter, and $Z_{K}(x)$ and $v_{l_{1}}(x)\left(K=1,2, \ldots, t ; l_{1}=1,2, \ldots, Q\right)$ are real-valued functions representing the fractional objective functions in crisp nature and the constraints, respectively. The numerator and denominator functions $f_{K}(x)$ and $g_{K}(x)(K=1,2, \ldots, t)$ are functions of real-valued also. The above problem gives feasible solutions only if $g_{K}(x)>0(K=1,2, \ldots, t)$.

\subsection{Linearization process for MFPP}

In this subsection, we describe the linearization process of MFPP which is based on Charnes and Cooper [6] transformation technique. Let us consider the transformation $s=1 / g_{K}(K=1,2, \ldots, t)$ and $z_{i j}=s x_{i j}(i=1,2, \ldots, m ; j=1,2, \ldots, n)$, where $s>0$ to transform MFPP (i.e., Model 2) into its linear version as given below:

\section{Model 2A}

$$
\begin{aligned}
\operatorname{minimize} & \left(s f_{K}\left(z_{i j} / s\right): K=1,2, \ldots, t\right) \\
\text { subject to } & s g_{K}\left(z_{i j} / s\right)=1 \quad(K=1,2, \ldots, t), \\
& v_{l_{1}}(x)\left(\begin{array}{l}
\leq \\
= \\
\geq
\end{array}\right) 0 \quad\left(l_{1}=1,2, \ldots, Q\right), \\
& x \geq 0 .
\end{aligned}
$$

Remark 3.1. If the optimization problem (i.e., Model 2) is of minimization type and structure is the same type of transportation problem (TP) then the equality constraints $s g_{K}\left(z_{i j} / s\right)=1 \quad(K=1,2, \ldots, t)$ of Model $2 \mathrm{~A}$ can be replaced by the inequality constraints $s g_{K}\left(z_{i j} / s\right) \geq 1(K=1,2, \ldots, t)$.

For the illustration of Remark 3.1, two examples are provided in below subsequently.

\section{Example 3.2.}

$$
\begin{aligned}
\text { minimize } & Z_{1}=\frac{36 z_{11}+31 z_{12}+12 z_{13}+6 z_{21}+27 z_{22}+38 z_{23}}{14 z_{11}+25 z_{12}+13 z_{13}+8 z_{21}+15 z_{22}+26 z_{23}} \\
\text { subject to } & z_{11}+z_{12}+z_{13} \leq 62, \\
& z_{21}+z_{22}+z_{23} \leq 75, \\
& z_{11}+z_{21} \geq 42,
\end{aligned}
$$


TABLE 2. Optimal solutions of two examples.

\begin{tabular}{lcc}
\hline \hline $\begin{array}{l}\text { Type of constraint/ } \\
\text { constraints }\end{array}$ & $\begin{array}{c}\text { Optimal values of } Z_{1} \\
\text { for Example } 3.2\end{array}$ & $\begin{array}{c}\text { Optimal values of } Z_{1} \\
\text { and } Z_{2} \text { for Example } 3.3\end{array}$ \\
\hline $\begin{array}{l}\text { Using equality constraint/ } \\
\text { constraints }\end{array}$ & 1.09 & $0.48,0.31$ \\
$\begin{array}{l}\text { Using inequality constraint/ } \\
\text { constraints (" } \geq \text { ") }\end{array}$ & 1.09 & $0.47,0.31$ \\
\hline
\end{tabular}

$$
\begin{aligned}
& z_{12}+z_{22} \geq 28 \\
& z_{13}+z_{23} \geq 58, \\
& z_{i j} \geq 0 \forall i, \quad j .
\end{aligned}
$$

\section{Example 3.3.}

$$
\begin{aligned}
\text { minimize } & Z_{1}=\frac{4 z_{11}+7 z_{12}+7 z_{13}+9 z_{21}+11 z_{22}+8 z_{23}}{9 z_{11}+15 x_{12}+13 z_{13}+18 z_{21}+19 z_{22}+17 z_{23}} \\
\text { minimize } & Z_{2}=\frac{2 z_{11}+4 z_{12}+3 z_{13}+5 z_{21}+8 z_{22}+7 z_{23}}{13 z_{11}+15 x_{12}+18 z_{13}+15 z_{21}+18 z_{22}+19 z_{23}} \\
\text { subject to } & z_{11}+z_{12}+z_{13} \leq 26 \\
& z_{21}+z_{22}+z_{23} \leq 24 \\
& z_{11}+z_{21} \geq 15 \\
& z_{12}+z_{22} \geq 16 \\
& z_{13}+z_{23} \geq 12 \\
& z_{i j} \geq 0 \forall i, j .
\end{aligned}
$$

To find the optimal solution from the above two examples, it is needed to convert the non-linear equations into their linear form. So, the transformation $y_{i j}=s z_{i j}(i=1,2 ; j=1,2,3)$, where $s>0$ (introduced by Charnes and Cooper [6]) is used to transform the non-linear equations into their linear form. Thereafter, the examples are solved by Lingo optimizer. It is also mentioned that the authors of [6] had provided a theorem on the optimality of fractional model. The solutions are displayed in Table 2.

It is observed from Table 2 that the objective functions values are either equal or better values if we replace "equality constraints" by "inequality constraints $(\geq)$ " for minimization problem. Thus, in the minimization problem, the obtained objective value of using "inequality constraints" should be equal to or less than that of using "equality constraints" due to the boundary feasible domain. Therefore, our observation, it is more acceptable to replace "equality constraints" by "inequality constraints $(\geq)$ " for solving a multi-objective minimization problem of TP types.

\subsection{The fuzzy-MFFTP}

Herein, we design the mathematical model of fuzzy MFFTP. The first objective function (i.e., the ratio of total cost/investment), other objective functions and capacity of supply center, and demand center are presented as fuzzy numbers. The mathematical model of the fuzzy MFFTP (i.e., Model 3) is formulated as stated subsequently. 


\section{Model 3}

$$
\begin{aligned}
\text { minimize } & \left(\tilde{Z}_{K}: K=1,2, \ldots, t\right) \\
& \text { or }\left(\frac{\tilde{z}_{K}(x)}{\tilde{g}_{K}(x)}: K=1,2, \ldots, t\right), \\
& \text { or }\left[\frac{\sum_{i=1}^{m} \sum_{j=1}^{n}\left[\tilde{z}_{1}\left(x_{i j}, \eta\left(x_{i j}\right)\right)\right]}{\sum_{i=1}^{m} \sum_{j=1}^{n}\left[\tilde{g}_{1}\left(x_{i j}\right)\right]},\right. \\
& \left.\frac{\sum_{i=1}^{m} \sum_{j=1}^{n}\left[\tilde{z}_{K}\left(x_{i j}\right)\right]}{\sum_{i=1}^{m} \sum_{j=1}^{n}\left[\tilde{g}_{K}\left(x_{i j}\right)\right]}: K=2,3, \ldots, t\right] \\
\text { subject to } & \sum_{j=1}^{n} x_{i j} \leq \tilde{a}_{i} \quad(i=1,2, \ldots, m), \\
& \sum_{i=1}^{m} x_{i j} \geq \tilde{b}_{j} \quad(j=1,2, \ldots, n), \\
& x_{i j} \geq 0(i=1,2, \ldots, m ; j=1,2, \ldots, n), \\
& \eta\left(x_{i j}\right)=\left\{\begin{array}{l}
0 \text { if } x_{i j}=0, \\
1 \text { if } x_{i j}>0 .
\end{array}\right.
\end{aligned}
$$

Here, $\tilde{z}_{K}$ and $\tilde{g}_{K}$ being the $K$ th fuzzy objective functions in the numerator and denominator respectively, and $\tilde{a}_{i}$ and $\tilde{b}_{j}$ the fuzzy capacity of source $i$ and fuzzy demand by destination $j$, again, respectively. As the objective functions $\left(\tilde{Z}_{K}\right)$ in fuzzy nature are of fractional forms, so we minimize the objective functions in the numerator, i.e., $\tilde{z}_{1}\left(x_{i j}, y_{i j}\right), \tilde{z}_{2}\left(x_{i j}\right), \ldots, \tilde{z}_{t}\left(x_{i j}\right)$, and the objective functions in denominator are converted to the inequality constraints, i.e., $\tilde{g}_{1}\left(x_{i j}\right) \geq 1, \tilde{g}_{2}\left(x_{i j}\right) \geq 1, \ldots, \tilde{g}_{t}\left(x_{i j}\right) \geq 1$. Feasibility criterion of Model 3 is stated as below.

$$
\sum_{i=1}^{m} \tilde{a}_{i} \gtrsim \sum_{j=1}^{n} \tilde{b}_{j},
$$

where by " $\gtrsim "$ we mean the fuzzy inequality.

\subsection{The fuzzy linear MFCTP}

This subsection describes the transformation of fuzzy MFFTP (i.e., Model 3) to fuzzy linear MFCTP (i.e., Model 4). Based on Remark 3.1 and using the transformation $z_{i j}=s x_{i j}[6] \quad(i=1,2, \ldots, m ; j=1,2, \ldots, n)$, where $s>0$, Model 3 is converted in such a way that the values of $\tilde{Z}_{K}(K=1,2, \ldots, t)$, are minimized. The equivalent fuzzy linear model of fuzzy MFFTP is designed in the following way.

\section{Model 4}

$$
\begin{aligned}
\operatorname{minimize} & \left(s \tilde{z}_{K}\left(z_{i j} / s\right): K=1,2, \ldots, t\right) \\
& \text { or }\left[\sum_{i=1}^{m} \sum_{j=1}^{n} s\left[\tilde{z}_{1}\left(z_{i j} / s, \eta\left(z_{i j} / s\right)\right)\right],\right. \\
& \left.\sum_{i=1}^{m} \sum_{j=1}^{n} s\left[\tilde{z}_{K}\left(z_{i j} / s\right): K=2,3, \ldots, t\right]\right] \\
\text { subject to } & \frac{1}{s} \sum_{j=1}^{n} z_{i j} \leq \tilde{a}_{i} \quad(i=1,2, \ldots, m),
\end{aligned}
$$




$$
\begin{gathered}
\frac{1}{s} \sum_{i=1}^{m} z_{i j} \geq \tilde{b}_{j} \quad(j=1,2, \ldots, n), \\
s\left[\sum_{i=1}^{m} \sum_{j=1}^{n} \tilde{g}_{1}\left(z_{i j} / s\right)\right] \geq 1, \\
s\left[\sum_{i=1}^{m} \sum_{j=1}^{n} \tilde{g}_{2}\left(z_{i j} / s\right)\right] \geq 1, \\
\vdots \\
s\left[\sum_{i=1}^{m} \sum_{j=1}^{n} \tilde{g}_{t}\left(z_{i j} / s\right)\right] \geq 1, \\
s>0, z_{i j} \geq 0, \forall i, j, \\
\eta\left(z_{i j} / s\right)=\left\{\begin{array}{l}
0 \text { if } z_{i j}=0, \\
1 \text { if } z_{i j}>0 .
\end{array}\right.
\end{gathered}
$$

Remark 3.4. An exact number " $a$ " can be equivalently presented a fuzzy number $(a, a, a)$, so we can claim that a linear FPP with crisp parameters is a special case of linear FPP with parameters are triangular fuzzy number in nature.

Many events arise in the practical situations in which the coefficients in a linear FPP are not exact when they are modeled mathematically. So, it is more acceptable to treat the coefficients as the triangular fuzzy numbers instead of crisp numbers. In this regard and based on Remark 3.4, it is said that Model 4 is an extended and special version of the classical linear FPP.

\section{THE FUZZY MFCTP IN DETERMINISTIC FORM}

Model 4 is a theoretical model than a realistic one because

1. it is not possible to minimize an imprecise quantity, like fuzzy cost $\tilde{z}_{1}$ and other fuzzy objective functions $\tilde{z}_{2}, \tilde{z}_{3}, \ldots, \tilde{z}_{t}$, and

2. constraints of Model 4 are not a crisp feasible set.

So, it is required to transform the fuzzy MFCTP into an identical deterministic model. In order to convert Model 4 into deterministic form, we adopt two techniques and discuss later subsection, namely,

- Robust Ranking, and

- Fuzzy Chance-Constrained Rough Approximation.

\subsection{Robust ranking technique}

RR technique was originated by Yager [54], it follows the compensation, linearity and additive properties. Robust ranking, $\mathcal{R}$ for a triangular fuzzy number $\tilde{c}$ is defined as below.

$$
\mathcal{R}(\tilde{c})=\int_{0}^{1}\left[\frac{c_{L}(\gamma)+c_{R}(\gamma)}{2}\right] d \gamma
$$

where $\left[c_{L}(\gamma), c_{R}(\gamma)\right]$ represents the $\gamma$-level cut of $\tilde{c}$.

Robust ranking technique for fuzzy objective function gives the crisp value which represents the average value of the fuzzy objective function. To convert fuzzy MFCTP (i.e., Model 4) into its similar crisp form, we leverage 
on the robust ranking technique and formulate our new model (i.e., Model 5) is as below.

\section{Model 5}

$$
\begin{aligned}
\operatorname{minimize} & \left(\mathcal{R}\left(s \tilde{z}_{K}\left(z_{i j} / s\right)\right): K=1,2, \ldots, t\right) \\
& \text { or }\left[\sum_{i=1}^{m} \sum_{j=1}^{n} s\left[\mathcal{R}\left(\tilde{z}_{1}\right)\left(z_{i j} / s, \eta\left(z_{i j} / s\right)\right)\right]\right. \\
& \left.\sum_{i=1}^{m} \sum_{j=1}^{n} s\left[\mathcal{R}\left(\tilde{z}_{K}\right)\left(z_{i j} / s\right): K=2,3, \ldots, t\right]\right] \\
\text { subject to } & \frac{1}{s} \sum_{j=1}^{n} z_{i j} \leq \mathcal{R}\left(\tilde{a}_{i}\right) \quad(i=1,2, \ldots, m), \\
& \frac{1}{s} \sum_{i=1}^{m} z_{i j} \geq \mathcal{R}\left(\tilde{b}_{j}\right) \quad(j=1,2, \ldots, n), \\
& s\left[\sum_{i=1}^{m} \sum_{j=1}^{n} \mathcal{R}\left(\tilde{g}_{1}\right)\left(z_{i j} / s\right)\right] \geq 1, \\
& s\left[\sum_{i=1}^{m} \sum_{j=1}^{n} \mathcal{R}\left(\tilde{g}_{2}\right)\left(z_{i j} / s\right)\right] \geq 1, \\
& \vdots \\
& s\left[\sum_{i=1}^{m} \sum_{j=1}^{n} \mathcal{R}\left(\tilde{g}_{t}\right)\left(z_{i j} / s\right)\right] \geq 1, \\
& s>0, z_{i j} \geq 0, \forall i, j, \\
& \eta\left(z_{i j} / s\right)=\left\{\begin{array}{l}
0 \text { if } z_{i j}=0 \\
1 \text { if } z_{i j}>0 .
\end{array}\right. \\
&
\end{aligned}
$$

Again, this model will be feasible, if and only if

$$
\sum_{i=1}^{m} \mathcal{R}\left(\tilde{a}_{i}\right) \geq \sum_{j=1}^{n} \mathcal{R}\left(\tilde{b}_{j}\right) .
$$

Definition 4.1. A feasible solution $y^{*}=\left(y_{i j}^{*}: i=1,2, \ldots, m ; j=1,2, \ldots, n\right)$ of Model 5 is called a non-dominated (Pareto-optimal) solution if there does not exist another feasible solution $y=\left(y_{i j}: i=\right.$ $1,2, \ldots, m ; j=1,2, \ldots, n)$ such that

$$
\begin{aligned}
& \mathcal{R}\left[\tilde{z}_{K}(y)\right] \leq \mathcal{R}\left[\tilde{z}_{K}\left(y^{*}\right)\right] \quad \forall K, \text { and } \\
& \mathcal{R}\left[\tilde{z}_{K}(y)\right]<\mathcal{R}\left[\tilde{z}_{K}\left(y^{*}\right)\right] \text { for at least one } K=1,2, \ldots, t .
\end{aligned}
$$

\subsection{Fuzzy chance-constrained programming}

Fuzzy chance-constrained programming (FCP) [23] is used to tackle fuzzy parameters involving in the objective functions as well as in the constraints. To deal with our fuzzy-MFCTP (Model 4), first we formulate an FCP version of Model 4. After that, using different fuzzy scales (i.e., Pos, Nec and Cr), is hence, we convert the deterministic model of Model 4. Therefore, a fuzzy chance-constrained version of Model 4 (i.e., Model 6) is formulated as: 


\section{Model 6}

$$
\begin{array}{ll}
\text { minimize } & \left(\overline{\tilde{z}}_{K}: K=1,2, \ldots, t\right) \\
\text { subject to } & \operatorname{Ch}\left\{s \tilde{z}_{K}\left(z_{i j} / s\right) \leq \overline{\tilde{z}}_{K}\right\} \geq \delta_{K}, \\
& \operatorname{Ch}\left\{\frac{1}{s} \sum_{j=1}^{n} z_{i j}-\tilde{a}_{i} \leq 0\right\} \geq \theta_{m_{i}} \quad(i=1,2, \ldots, m), \\
& \operatorname{Ch}\left\{\frac{1}{s} \sum_{i=1}^{m} z_{i j}-\tilde{b}_{j} \geq 0\right\} \geq \theta_{n_{j}} \quad(j=1,2, \ldots, n), \\
& \operatorname{Ch}\left\{s\left[\sum_{i=1}^{m} \sum_{j=1}^{n} \tilde{g}_{1}\left(z_{i j} / s\right)\right]-1=0\right\} \geq \theta_{t_{1}}, \\
& \operatorname{Ch}\left\{s\left[\sum_{i=1}^{m} \sum_{j=1}^{n} \tilde{g}_{2}\left(z_{i j} / s\right)\right]-1=0\right\} \geq \theta_{t_{2}}, \\
& \vdots \quad \\
& \operatorname{Ch}\left\{s\left[\sum_{i=1}^{m} \sum_{j=1}^{n} \tilde{g}_{t}\left(z_{i j} / s\right)\right]-1=0\right\} \geq \theta_{t_{t}}, \\
& s>0, z_{i j} \geq 0, \forall i, j, \\
& \eta\left(z_{i j} / s\right)=\left\{\begin{array}{l}
0 \text { if } z_{i j}=0, \\
1 \text { if } z_{i j}>0 .
\end{array}\right.
\end{array}
$$

Here, Ch symbolizes fuzzy scale, i.e., Pos, Nec and $\mathrm{Cr}$, and $\delta_{K}(K=1,2, \ldots, t)$ and $\theta_{r}$ $(r=1,2, \ldots, m, n, t ; m \neq n \neq t)$ are the predetermined confidence levels for the objective functions and the constraints respectively. So, $\left(\theta_{m_{i}}, \theta_{n_{j}}, \theta_{t_{K}}\right) \in \theta_{r}$. As the problem is of minimization type, we minimize the objective functions $\overline{\tilde{z}}_{1}, \overline{\tilde{z}}_{2}, \ldots, \overline{\tilde{z}}_{t}$, satisfying the chance-constrained levels.

Remark 4.2. To formulate fuzzy chance-constrained rough model of Model 6, we assume feasible region $D=\left\{x: x \in X, \operatorname{Ch}\left\{s \tilde{z}_{K}\left(z_{i j} / s\right) \leq \overline{\tilde{z}}_{K}\right\} \geq \delta_{K}(K=1,2, \ldots, t)\right.$ and $\operatorname{Ch}\left\{q_{r}\left(z_{i j} / s\right) \geq 0\right\} \geq \theta_{r}(r=$ $1,2, \ldots, m, n, t ; m \neq n \neq t)\}$, where $q_{r}$ represents the set of constraints. $\overline{\tilde{z}}_{K}$ is the smallest possible value of $\tilde{z}_{K}$. Two sets $\mathrm{L}$ and $\mathrm{U}$ are constructed and defined in the following manner.

$$
\begin{aligned}
L= & \left\{x: x \in X, \operatorname{Nec}\left\{s \tilde{z}_{K}\left(z_{i j} / s\right) \leq \overline{\tilde{z}}_{K}\right\} \geq \delta_{K}(K=1,2, \ldots, t)\right. \text { and } \\
& \left.\operatorname{Nec}\left\{q_{r}\left(z_{i j} / s\right) \geq 0\right\} \geq \theta_{r}\right\}, \\
U= & \left\{x: x \in X, \operatorname{Pos}\left\{s \tilde{z}_{K}\left(z_{i j} / s\right) \leq \overline{\tilde{z}}_{K}\right\} \geq \delta_{K}(K=1,2, \ldots, t)\right. \text { and } \\
& \left.\operatorname{Pos}\left\{q_{r}\left(z_{i j} / s\right) \geq 0\right\} \geq \theta_{r}\right\}
\end{aligned}
$$

where $r=1,2, \ldots, m, n, t ; m \neq n \neq t$.

Theorem 4.3. For the feasible region $D$; we have the relation, $L \subseteq D \subseteq U$, where $L$ and $U$ are already defined as above.

Proof. For any $\left(z_{0} / s\right) \in X$, if $x_{0} \in L$, i.e.,

$\operatorname{Nec}\left\{s \tilde{z}_{K}\left(z_{0} / s\right) \leq \overline{\tilde{z}}_{K}\right\} \geq \delta_{K}(K=1,2, \ldots, t)$ and $\operatorname{Nec}\left\{q_{r}\left(z_{0} / s\right) \geq\right.$ or $\left.\leq 0\right\} \geq \theta_{r}(r=1,2, \ldots, m, n, t ; m \neq$ $n \neq t)$. From Theorem 2.12 it can be written as $\operatorname{Cr}\left\{s \tilde{z}_{K}\left(z_{0} / s\right) \leq \overline{\tilde{z}}_{K}\right\} \geq \operatorname{Nec}\left\{s \tilde{z}_{K}\left(z_{0} / s\right) \leq \overline{\tilde{z}}_{K}\right\} \geq \delta_{K}(K=$ 
$1,2, \ldots, t)$ and $\operatorname{Cr}\left\{q_{r}\left(z_{0} / s\right) \geq\right.$ or $\left.\leq 0\right\} \geq \operatorname{Nec}\left\{q_{r}\left(z_{0} / s\right) \geq\right.$ or $\left.\leq 0\right\} \geq \theta_{r},(r=1,2, \ldots, m, n, t ; m \neq n \neq t)$, i.e., $\left(z_{0} / s\right) \in D$. Thus, $L \subseteq D$. In the same way, it concludes that $D \subseteq U$.

Hence, the assertion of the theorem follows.

Let us consider $L=\underline{D}$ and $U=\bar{D}$; using $\underline{D}$ and $\bar{D}$ to approximate $D$. It is obvious that $\underline{D} \subseteq D \subseteq \bar{D}$. Then, Model 6 can be converted into Model 7 and Model 8.

\subsection{The fuzzy chance-constrained rough MFCTP}

Here, we extend the feasible region of Model 6 using rough approximation technique. Therefore, we transform Model 6 into two models: the LAM of our fuzzy chance-constrained rough MFCTP, namely, LA-MFCTP, (i.e., Model 7) is presented as:

\section{Model 7}

$$
\begin{aligned}
\operatorname{minimize} & \left(\overline{\tilde{z}}_{K}: K=1,2, \ldots, t\right) \\
\text { subject to } & \operatorname{Nec}\left\{s \tilde{z}_{K}\left(z_{i j} / s\right) \leq \overline{\tilde{z}}_{K}\right\} \geq \delta_{K} \\
& \operatorname{Nec}\left\{\frac{1}{s} \sum_{j=1}^{n} z_{i j}-\tilde{a}_{i} \leq 0\right\} \geq \theta_{m_{i}} \quad(i=1,2, \ldots, m), \\
& \operatorname{Nec}\left\{\frac{1}{s} \sum_{i=1}^{m} z_{i j}-\tilde{b}_{j} \geq 0\right\} \geq \theta_{n_{j}} \quad(j=1,2, \ldots, n), \\
& \operatorname{Nec}\left\{s\left[\sum_{i=1}^{m} \sum_{j=1}^{n} \tilde{g}_{1}\left(z_{i j} / s\right)\right]-1 \geq 0\right\} \geq \theta_{t_{1}}, \\
& \operatorname{Nec}\left\{s\left[\sum_{i=1}^{m} \sum_{j=1}^{n} \tilde{g}_{2}\left(z_{i j} / s\right)\right]-1 \geq 0\right\} \geq \theta_{t_{2}}, \\
& \vdots \\
& \operatorname{Nec}\left\{s\left[\sum_{i=1}^{m} \sum_{j=1}^{n} \tilde{g}_{t}\left(z_{i j} / s\right)\right]-1 \geq 0\right\} \geq \theta_{t_{t}}, \\
& s>0, z_{i j} \geq 0, \forall i, j, \\
& \eta\left(z_{i j} / s\right)=\left\{\begin{array}{l}
0 \text { if } z_{i j}=0, \\
1 \text { if } z_{i j}>0,
\end{array}\right.
\end{aligned}
$$

and the UAM of our fuzzy chance-constrained rough MFCTP, namely, UA-MFCTP, (i.e., Model 8) is formulated as follows:

\section{Model 8}

$\operatorname{minimize}\left(\overline{\tilde{z}}_{K}: K=1,2, \ldots, t\right)$

subject to $\operatorname{Pos}\left\{s \tilde{z}_{K}\left(z_{i j} / s\right) \leq \overline{\tilde{z}}_{K}\right\} \geq \delta_{K}$,

$$
\operatorname{Pos}\left\{\frac{1}{s} \sum_{j=1}^{n} z_{i j}-\tilde{a}_{i} \leq 0\right\} \geq \theta_{m_{i}} \quad(i=1,2, \ldots, m),
$$




$$
\begin{aligned}
& \operatorname{Pos}\left\{\frac{1}{s} \sum_{i=1}^{m} z_{i j}-\tilde{b}_{j} \geq 0\right\} \geq \theta_{n_{j}} \quad(j=1,2, \ldots, n), \\
& \operatorname{Pos}\left\{s\left[\sum_{i=1}^{m} \sum_{j=1}^{n} \tilde{g}_{1}\left(z_{i j} / s\right)\right]-1 \geq 0\right\} \geq \theta_{t_{1}}, \\
& \operatorname{Pos}\left\{s\left[\sum_{i=1}^{m} \sum_{j=1}^{n} \tilde{g}_{2}\left(z_{i j} / s\right)\right]-1 \geq 0\right\} \geq \theta_{t_{2}}, \\
& \vdots \\
& \operatorname{Pos}\left\{s\left[\sum_{i=1}^{m} \sum_{j=1}^{n} \tilde{g}_{t}\left(z_{i j} / s\right)\right]-1 \geq 0\right\} \geq \theta_{t_{t}}, \\
& s>0, z_{i j} \geq 0, \forall i, j, \\
& \eta\left(z_{i j} / s\right)=\left\{\begin{array}{l}
0 \text { if } z_{i j}=0, \\
1 \text { if } z_{i j}>0 .
\end{array}\right.
\end{aligned}
$$

Definition 4.4. A feasible solution at $\theta_{r}$-necessity level, $z_{i j}^{*}$, of Model 7 is called a $\delta_{K}$-efficient solution if there does not exist another feasible solution at $\theta_{r}$-necessity level $z_{i j}$ such that $\operatorname{Nec}\left\{s \quad \tilde{z}_{K}\left(z_{i j} / s\right)\right\} \geq \delta_{K}$ with $\tilde{z}_{K}\left(z_{i j}\right) \leq \overline{\tilde{z}}_{K}\left(z_{i j}^{*}\right)$ for all $K$ and $\tilde{z}_{K}\left(z_{i j}\right)<\overline{\tilde{z}}_{K}\left(z_{i j}^{*}\right)$ for at least one $K \in\{1,2, \ldots, t\}$.

Definition 4.5. A feasible solution at $\theta_{r}$-possibility level, $z_{i j}^{*}$, of Model 8 is called a $\delta_{K}$-efficient solution if there does not exist another feasible solution at $\theta_{r}$-possibility level $z_{i j}$ such that $\operatorname{Pos}\left\{s \tilde{z}_{K}\left(z_{i j} / s\right)\right\} \geq \delta_{K}$ with $\tilde{z}_{K}\left(z_{i j}\right) \leq \overline{\tilde{z}}_{K}\left(z_{i j}^{*}\right)$ for all $K$ and $\tilde{z}_{K}\left(z_{i j}\right)<\overline{\tilde{z}}_{K}\left(z_{i j}^{*}\right)$ for at least one $K \in\{1,2, \ldots, t\}$.

Theorem 4.6. Let $\tilde{c}_{i j}$ be a triangular fuzzy number with membership function $\mu_{\tilde{c}_{i j}}(x)$, shown in Figure 1; it is defined as:

$$
\mu_{\tilde{c}_{i j}}(x)=\left\{\begin{array}{lll}
\frac{x-\left(c_{i j}-\alpha_{i j}^{c}\right)}{\alpha_{i j}^{c}}, & \text { if } & \left(c_{i j}-\alpha_{i j}^{c}\right) \leq x \leq c_{i j}, \\
\frac{\left(c_{i j}+\beta_{i j}^{i}\right)-x}{\beta_{i j}^{c}}, & \text { if } & c_{i j} \leq x \leq\left(c_{i j}+\beta_{i j}^{c}\right), \\
0, & \text { otherwise, } &
\end{array}\right.
$$

where $c_{i j}$ is a real number; $\alpha_{i j}^{c}$ and $\beta_{i j}^{c}$ are the left and right spreads of $\tilde{c}_{i j}(i=1,2, \ldots, m$;

$j=1,2, \ldots, n)$. If the reference function of $\tilde{c}_{i j}$ is $(1-w), w \in(0,1)$, then,

$\operatorname{Pos}\left\{\tilde{c}_{i j} x \leq \overline{\tilde{z}}_{K}\right\} \geq \delta_{K}$ is equivalent to $\overline{\tilde{z}}_{K} \geq c_{i j} x+\left(1-\delta_{K}\right) \beta_{i j}^{c} x(K=1,2, \ldots, t)$.

Proof. As $\tilde{c}_{i j}$ is a triangular fuzzy number and its membership function is $\mu_{\tilde{c}_{i j}}$. By extension principle of Zadeh [56], the membership function of fuzzy number $\mu_{\tilde{c}_{i j}}(x)$ is considered as:

$$
\mu_{\tilde{c}_{i j}}(x(u))=\left\{\begin{array}{lll}
\frac{u-\left(c_{i j} x-\alpha_{i j}^{c} x\right)}{\alpha_{i j}^{c} x}, & \text { if } \quad\left(c_{i j} x-\alpha_{i j}^{c} x\right) \leq u \leq c_{i j} x, \\
\frac{\left(c_{i j} x+\beta_{i j}^{c} x\right)-u}{\beta_{i j}^{c} x}, & \text { if } \quad c_{i j} x \leq u \leq\left(c_{i j} x+\beta_{i j}^{c} x\right), \\
0, \quad \text { otherwise, } &
\end{array}\right.
$$

for $i=1,2, \ldots, m ; j=1,2, \ldots, n$. For simplicity, we denote the triangular fuzzy number $\tilde{c}_{i j}=\left(c_{i j}, \alpha_{i j}^{c}, \beta_{i j}^{c}\right)$; then according to Lemma 2.11, we can write

$$
\operatorname{Pos}\left\{\tilde{c}_{i j} x \leq \overline{\tilde{z}}_{K}\right\} \geq \delta_{K} \Leftrightarrow \overline{\tilde{z}}_{K} \geq c_{i j} x+\left(1-\delta_{K}\right) \beta_{i j}^{c} x \quad(K=1,2, \ldots, t) .
$$

Hence, the assertion of our theorem results. 


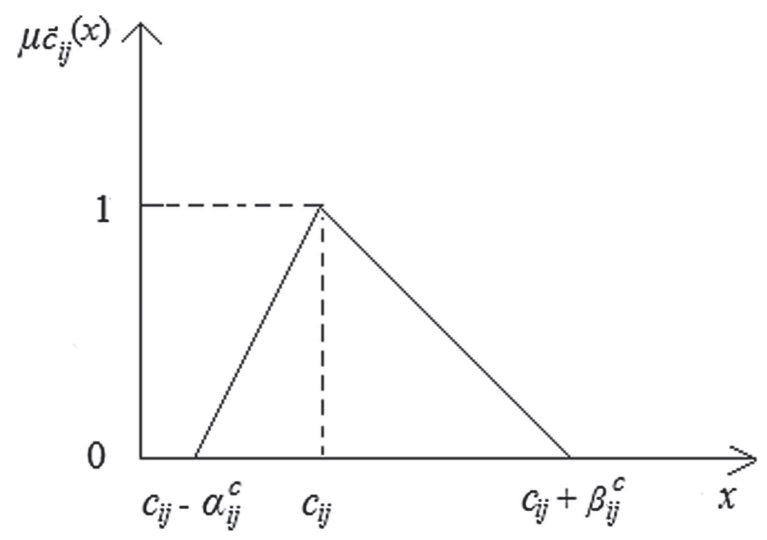

FIGURE 1. Pictorial representation of a triangular fuzzy number.

Theorem 4.7. Let $\tilde{p}_{i j}$ and $\tilde{h}_{r}$ be the triangular fuzzy numbers with membership functions are depicted in the following way, respectively:

$$
\mu_{\tilde{p}_{i j}}(x)=\left\{\begin{array}{lll}
\frac{x-\left(p_{i j}-\alpha_{i j}^{p}\right)}{\alpha_{i j}^{p}}, & \text { if } & \left(p_{i j}-\alpha_{i j}^{p}\right) \leq x \leq p_{i j}, \\
\frac{\left(p_{i j}+\beta_{i j}^{p}\right)-x}{\beta_{i j}^{p}}, & \text { if } & p_{i j} \leq x \leq\left(p_{i j}+\beta_{i j}^{p}\right), \\
0, & \text { otherwise, } &
\end{array}\right.
$$

and

$$
\mu_{\tilde{h}_{r}}(x)=\left\{\begin{array}{lll}
\frac{x-\left(h_{r}-\alpha_{r}^{h}\right)}{\alpha_{r}^{h}}, & \text { if } & \left(h_{r}-\alpha_{r}^{h}\right) \leq x \leq h_{r}, \\
\frac{\left(h_{r}+\beta_{r}^{h}\right)-x}{\beta_{r}^{h}}, & \text { if } & h_{r} \leq x \leq\left(h_{r}+\beta_{r}^{h}\right), \\
0, & \text { otherwise, }
\end{array}\right.
$$

where $p_{i j}$ and $h_{r}$ are real numbers; $\alpha_{i j}^{p}$ and $\beta_{i j}^{p}$ are the left and right spreads of $\tilde{p}_{i j}, \alpha_{r}^{h}$ and $\beta_{r}^{h}$ are the left and right spreads of $\tilde{h}_{r}(i=1,2, \ldots, m ; j=1,2, \ldots, n ; r=1,2, \ldots, m, n, t ; m \neq n \neq t)$. If the reference function of $\tilde{p}_{i j}$ and $\tilde{h}_{r}$ is $(1-w)$ for some $w \in(0,1)$ and we suppose that $p_{i j}$ and $h_{r}$ are independent. Then, $\operatorname{Pos}\left\{\tilde{p}_{i j} x \geq \tilde{h}_{r}\right\} \geq \theta_{r}$ is equivalent to $h_{r}+\left(1-\theta_{r}\right) \beta_{r}^{h} \leq p_{i j} x+\left(1-\theta_{r}\right) \beta_{i j}^{p} x, \quad r=1,2, \ldots, m, n, t ; m \neq n \neq t$.

Proof. As $\tilde{p}_{i j}$ is a triangular fuzzy number and its membership function is $\mu_{\tilde{p}_{i j}}$. From extension principle of Zadeh [56], the membership function of fuzzy number $\mu_{\tilde{p}_{i j}}(x)$ is specified as:

$$
\mu_{\tilde{p}_{i j}}(x(u))=\left\{\begin{array}{lll}
\frac{u-\left(p_{i j} x-\alpha_{i j}^{p} x\right)}{\alpha_{i j}^{p} x}, & \text { if } \quad\left(p_{i j} x-\alpha_{i j}^{p} x\right) \leq u \leq p_{i j} x, \\
\frac{\left(p_{i j} x+\beta_{i j}^{p} x\right)-u}{\beta_{i j}^{p} x}, & \text { if } \quad & p_{i j} x \leq u \leq\left(p_{i j} x+\beta_{i j}^{p} x\right), \\
0, \quad \text { otherwise, } &
\end{array}\right.
$$

for $i=1,2, \ldots, m ; j=1,2, \ldots, n$; and $\tilde{h}_{r}(r=1,2, \ldots, m, n, t ; m \neq n \neq t)$ is also a triangular fuzzy number with membership function $\mu_{\tilde{h}_{r}}$. Without any loss of generality, we represent triangular fuzzy number $\tilde{p}_{i j}=\left(p_{i j}, \alpha_{i j}^{p}, \beta_{i j}^{p}\right)$ and $\tilde{h}_{r}=\left(h_{r}, \alpha_{r}^{h}, \beta_{r}^{h}\right)$. Then, using Lemma 2.11, we obtain $\operatorname{Pos}\left\{\tilde{p}_{i j} x \geq \tilde{h}_{r}\right\} \geq \theta_{r} \Leftrightarrow h_{r}-\theta_{r} \alpha_{r}^{h} \leq p_{i j} x+\left(1-\theta_{r}\right) \beta_{i j}^{p} x \quad(r=1,2, \ldots, m, n, t ; m \neq n \neq t)$.

Hence, the proof of our theorem follows.

Theorem 4.8. Considering that the fuzzy numbers are same as those in Theorem 4.6. Then, Nec $\left\{\tilde{c}_{i j} x \leq \overline{\tilde{z}}_{K}\right\} \geq$ $\delta_{K}$ is equivalent to $\overline{\tilde{z}}_{K} \geq c_{i j} x-\delta_{K} \alpha_{i j}^{c} x \quad(K=1,2, \ldots, t)$. 
Proof. Refer to the proof of Theorem 4.6.

Theorem 4.9. Assuming that the fuzzy numbers are same as those in Theorem 4.7. Then, Nec\{ $\left\{\tilde{p}_{i j} x \geq \tilde{h}_{r}\right\} \geq \theta_{r}$ is equivalent to

$$
h_{r}+\left(1-\theta_{r}\right) \beta_{r}^{h} \leq p_{i j} x+\left(1-\theta_{r}\right) \beta_{i j}^{p} x \quad(r=1,2, \ldots, m, n, t ; m \neq n \neq t) .
$$

Proof. Refer to the proof of Theorem 4.7

\subsection{Deterministic rough version of the fuzzy-MFCTP}

Based on Nec and Pos measures and using Theorems 4.6, 4.7, 4.8 and 4.9, the equivalent deterministic models of Models 7 and 8 are stated as linear LAM of fuzzy chance-constrained rough MFCTP, namely, LLA-MFCTP (i.e., Model 9) is described as:

\section{Model 9}

$$
\begin{aligned}
\text { minimize } & \left(\overline{\tilde{z}}_{K}: K=1,2, \ldots, t\right) \\
\text { subject to } & \overline{\tilde{z}}_{1} \geq\left[\sum_{i=1}^{m} \sum_{j=1}^{n} s\left(c_{i j}\left(z_{i j} / s\right)-\delta_{1} \alpha_{i j}^{1}\left(z_{i j} / s\right)\right)+\left(f_{i j}\left(z_{i j} / s\right) \eta\left(z_{i j} / s\right)\right.\right. \\
& \left.\left.-\delta_{1} \alpha_{i j}^{f}\left(z_{i j} / s\right)\right)\right] \\
& \bar{z}_{2} \geq\left[\sum_{i=1}^{m} \sum_{j=1}^{n} s\left(z_{2}\left(z_{i j} / s\right)-\delta_{2} \alpha_{i j}^{2}\left(z_{i j} / s\right)\right)\right] \\
& \vdots \\
& \bar{z}_{t} \geq\left[\sum_{i=1}^{m} \sum_{j=1}^{n} s\left(z_{t}\left(z_{i j} / s\right)-\delta_{t} \alpha_{i j}^{t}\left(z_{i j} / s\right)\right)\right] \\
& \frac{1}{s} \sum_{j=1}^{n} z_{i j} \leq\left(a_{i}-\theta_{m_{i}} \alpha_{i}^{a}\right), \\
& \frac{1}{s} \sum_{i=1}^{m} z_{i j} \geq\left(b_{j}-\theta_{n_{j}} \alpha_{j}^{b}\right), \\
& s\left[\sum_{i=1}^{m} \sum_{j=1}^{n}\left[g_{1}\left(z_{i j} / s\right)+\left(1-\theta_{t_{1}}\right) \beta_{i j}^{1}\left(z_{i j} / s\right)\right] \geq 1,\right. \\
& s\left[\sum_{i=1}^{m} \sum_{j=1}^{n}\left[g_{2}\left(z_{i j} / s\right)+\left(1-\theta_{t_{2}}\right) \beta_{i j}^{2}\left(z_{i j} / s\right)\right] \geq 1,\right. \\
& \vdots \\
& s\left[\sum_{i=1}^{m} \sum_{j=1}^{n}\left[g_{t}\left(z_{i j} / s\right)+\left(1-\theta_{t_{t}}\right) \beta_{i j}^{t}\left(z_{i j} / s\right)\right] \geq 1,\right. \\
& \\
& \\
& \\
&
\end{aligned}
$$


and linear UAM of fuzzy chance-constrained rough MFCTP, namely, LUA-MFCTP (i.e., Model 10) is presented in the following way.

\section{Model 10}

$$
\begin{aligned}
& \operatorname{minimize}\left(\overline{\tilde{z}}_{K}: K=1,2, \ldots, t\right) \\
& \text { subject to } \overline{\tilde{z}}_{1} \leq\left[\sum_{i=1}^{m} \sum_{j=1}^{n} s\left(c_{i j}\left(z_{i j} / s\right)+\left(1-\delta_{1}\right) \beta_{i j}^{1}\left(z_{i j} / s\right)\right)+\left(f_{i j}\left(z_{i j} / s\right) \eta\left(z_{i j} / s\right)\right.\right. \\
&\left.\left.+\left(1-\delta_{1}\right) \beta_{i j}^{f}\left(z_{i j} / s\right)\right)\right] \\
& \overline{\tilde{z}}_{2} \leq\left[\sum_{i=1}^{m} \sum_{j=1}^{n} s\left(z_{2}\left(z_{i j} / s\right)+\left(1-\delta_{2}\right) \beta_{i j}^{2}\left(z_{i j} / s\right)\right)\right] \\
& \vdots \\
& \bar{z}_{t} \leq\left[\sum_{i=1}^{m} \sum_{j=1}^{n} s\left(z_{t}\left(z_{i j} / s\right)+\left(1-\delta_{t}\right) \beta_{i j}^{t}\left(z_{i j} / s\right)\right)\right] \\
& \frac{1}{s} \sum_{j=1}^{n} z_{i j} \leq\left(a_{i}+\left(1-\theta_{m_{i}}\right) \beta_{i}^{a}\right), \\
& \frac{1}{s} \sum_{i=1}^{m} z_{i j} \geq\left(b_{j}+\left(1-\theta_{n_{j}}\right) \beta_{j}^{b}\right), \\
& s\left[\sum_{i=1}^{m} \sum_{j=1}^{n}\left[g_{1}\left(z_{i j} / s\right)-\left(1-\theta_{t_{1}}\right) \alpha_{i j}^{1}\left(z_{i j} / s\right)\right] \geq 1\right. \\
& s\left[\sum_{i=1}^{m} \sum_{j=1}^{n}\left[g_{2}\left(z_{i j} / s\right)-\left(1-\theta_{t_{2}}\right) \alpha_{i j}^{2}\left(z_{i j} / s\right)\right] \geq 1\right. \\
& \vdots \\
& s\left[\sum_{i=1}^{m} \sum_{j=1}^{n}\left[g_{t}\left(z_{i j} / s\right)-\left(1-\theta_{t_{t}}\right) \alpha_{i j}^{t}\left(z_{i j} / s\right)\right] \geq 1\right. \\
& s>0, z_{i j} \geq 0, \forall i, j, \\
& \eta\left(z_{i j} / s\right)=\left\{0 \text { if } z_{i j}=0,\right. \\
& \text { if } z_{i j}>0 . \\
&
\end{aligned}
$$

A visual summary of models evolution is shown in Figure 2.

\section{DeFiCIENCIES OF EXISTING METHODS}

- Arya et al. [3] suggested a technique for solving fuzzy multiple objective FPP. From Table 6, it is observed that our proposed FCRA (Model 9: LLA-MFCTP) technique gives better optimal solutions than Arya et al.'s approach. Furthermore, we incorporate rough set approximation to extend as well partition the feasible region which is more acceptable to solve real-world optimization problem.

- Stanojević and Stanojević [44] found some mistakes of Chakraborty and Gupta's [7] approach to transform multi-objective FPP to its equivalent linear form and they pointed out the weakness of Chakraborty and Gupta's approach. However, our modified transformation procedure is free from such type of weakness. 


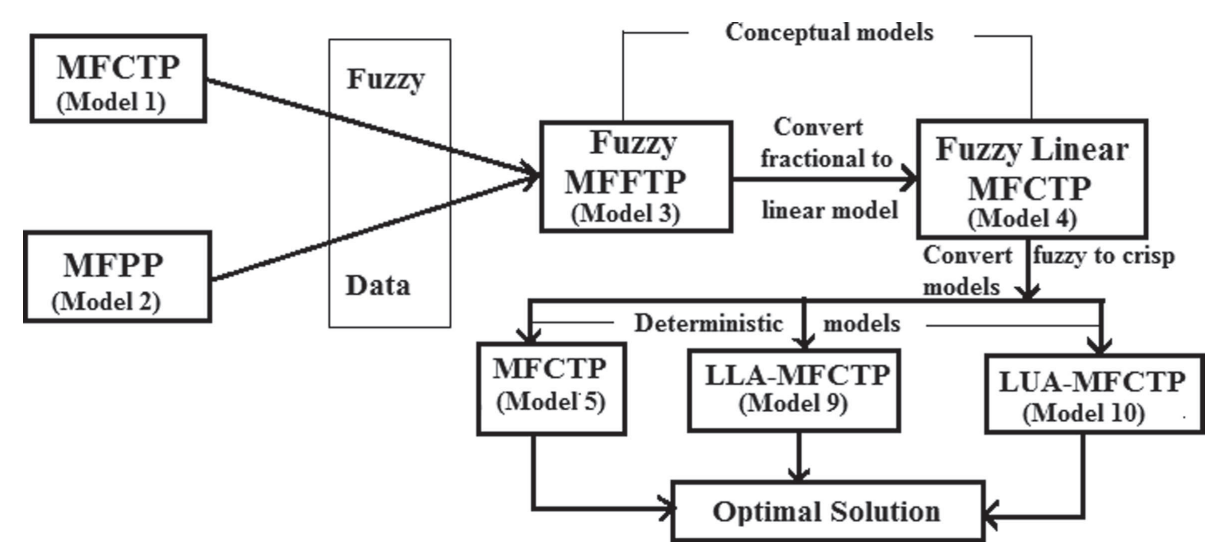

Figure 2. A visual summary of models evolution.

- Bhati and Singh [5] developed a branch and bound approach to derive solution of a multi-objective FPP. In their method, there exist several steps such as transforming maximization problem to minimization problem, converting multi-objective problem into single objective problem, calculating lower and upper bounds of the each objective function and others which lead more computational burden than our suggested FCRA technique to extract optimal solution from multi-objective FPP. Moreover, by adopting FCRA technique in the proposed MFFTP, we compute LA and UA optimal solutions of our formulated MFFTP which is more realistic than a single optimal solution of a practical optimization problem.

- Mahmoodirad et al. [25] formulated a linear fractional transportation problem under uncertain environment. They considered imprecise parameters in a transportation system as uncertain variable. But, uncertain variable is taken when historical information about data does not exist. So, a specified case arises which not happen every day transporting system. From this view, in the designed model the parameters are treated as triangular fuzzy number and rough approximation technique is imposed to extend optimal solution space. Furthermore, our designed model is formulated under multiple objective ground and an additional cost is taken as fixed-charge. Thus, our proposed model and FCRA technique are more reliable to tackle uncertainty in practical transportation problem than uncertain variable.

- Upmanyu and Saxena [47] extended a solution algorithm for solving multiple objective fractional fixed-charge problem. They solved the fractional problem without converting linear form. So, if the number of objective functions and variables in the objective functions are increased, then complexity arises in the computational process (i.e., to find optimal solution) whereas in our solution procedure has not been arisen any such types of complexity.

\section{The SOLUtion PROCEDURE}

To solve the deterministic models of the MFCTP (i.e., Models 5, 9 and 10), fuzzy programming method is applied which, based on [57], comprises the following steps:

Step 1: The MFCTP is solved by taking single objective function at a time and ignoring another. This process is repeated $K$ times for $K$ many different objective functions $(K=1,2, \ldots, t)$.

Step 2: The results of Step 1 are used to derive the similar value for each objective function and construct a pay-off matrix of format $K \times K$. Then, from the pay-off matrix, we find the lower bound $L^{K}$ and upper bound $U^{K}$ for the $K$ th objective function $z_{K}(K=1,2, \ldots, t)$, where $L^{K}$ are aspiration levels of achievement for the $K$ th objective, $U^{K}$ is the highest acceptable level of achievement for the $K$ th objective and $d^{K}=\left[U^{K}-L^{K}\right]$ is degradation allowance for the $K$ th objective function. 
Step 3: The best $\left(L^{K}\right)$ and worst $\left(U^{K}\right)$ for each objective function are calculated from Step 2. A primary fuzzy model are stated: find $z_{i j}(i=1,2, \ldots, m ; j=1,2, \ldots, n)$, so as to satisfy $z_{K} \leq L^{K}(K=1,2, \ldots, t)$ with the specified constraints and non-negative conditions. For the MFCTP, a membership function $\mu_{z_{K}}\left(z_{K}\right)$ corresponding to $K$ th objective function is presented as below.

$$
\mu_{z_{K}}\left(z_{K}\right)= \begin{cases}1, & \text { if } z_{K} \leq L^{K} \\ 1-\left(\frac{z_{K}-L^{K}}{U^{K}-L^{K}}\right), & \text { if } \quad L^{K}<z_{K}<U^{K} \quad(K=1,2, \ldots, t), \\ 0, & \text { if } z_{K} \geq U^{K}\end{cases}
$$

Step 4: Convert the fuzzy MFCTP, obtained in Step 3, into the following equivalent crisp model:

$$
\begin{aligned}
\operatorname{maximize} & \lambda \\
\text { subject to } & \lambda \leq \frac{U^{K}-z_{K}}{U^{K}-L^{K}} \quad(K=1,2, \ldots, t),
\end{aligned}
$$

with the same constraints of Model 5 or 9 or 10 and $\lambda \geq 0$,

$$
\text { where } \lambda=\min \left\{\mu_{z_{K}}\left(z_{K}\right): K=1,2, \ldots, t\right\} \text {. }
$$

The above problem can be written in a more simplified form as:

$$
\begin{aligned}
\operatorname{maximize} & \lambda \\
\text { subject to } & z_{K}+\lambda\left(U^{K}-L^{K}\right) \leq U^{K}(K=1,2, \ldots, t) \\
& \lambda \geq 0
\end{aligned}
$$

with the same constraints of Model 5 or 9 or 10 .

Theorem 6.1. If $y^{*}=\left(y_{i j}^{*}: i=1,2, \ldots, m ; j=1,2, \ldots, n\right)$ is defined as a non-dominated solution of Equation (6.1), then $y^{*}$ is a non-dominated solution of Model 5, i.e., there does not exist another feasible solution $y=\left(y_{i j}: i=1,2, \ldots, m ; j=1,2, \ldots, n\right)$ such that

$$
\begin{aligned}
& \mathcal{R}\left[\tilde{z}_{K}(y)\right] \leq \mathcal{R}\left[\tilde{z}_{K}\left(y^{*}\right)\right] \quad \forall K, \text { and } \\
& \mathcal{R}\left[\tilde{z}_{K}(y)\right]<\mathcal{R}\left[\tilde{z}_{K}\left(y^{*}\right)\right] \quad \text { for at least one } K=1,2, \ldots, t .
\end{aligned}
$$

Proof. This theorem is proved by contradiction. Assuming $y^{*}$ is a non-dominated solution of Model 5, i.e., there is a feasible solution $y$ such that the inequalities (6.2) and (6.3) are satisfied.

As $\mu_{K}(y)$ strictly decreases relative to the similar objective value $\mathcal{R}\left[\tilde{z}_{K}(y)\right]$ in $[0,1]$, we have $\mu_{K}(y) \geq$ $\mu_{K}\left(y^{*}\right) \forall K$, and $\mu_{K}(y)>\mu_{K}\left(y^{*}\right)$ for at least one $K=1,2, \ldots, t$.

Hence, it observes from strict monotonicity of the weight root-power mean operator [21] and using product min operator [57]; we that write $\mu_{K}(y)>\mu_{K}\left(y^{*}\right)$. Thus, a contradiction arises to the fact that $y^{*}$ is a nondominated solution of Equation (6.1). Hence, the theorem is proved.

Remark 6.2. Theorem 6.1 also holds for Models 9 and 10 also.

\subsection{Solution procedure for Model 5}

(1) Find the robust rank of each triangular fuzzy number which is described in Section 4.1. Obtain the crisp values for all fuzzy parameters of Model 5 .

(2) Solve this crisp MFCTP (Model 5) by using fuzzy programming which is described in Section 6 .

(3) Derive the optimal solution of each objective function (using, e.g., the LINGO, as in our case). 


\subsection{Solution procedure for Models 9 and 10}

(1) Set the confidence levels (choice of decision maker) of $\delta_{K}(K=1,2, \ldots, t)$ for objective functions and confidence levels of $\theta_{m_{i}}(i=1,2, \ldots, m), \theta_{n_{j}}(j=1,2, \ldots, n)$ and $\theta_{t_{K}}(K=1,2, \ldots, t)$ for constraints. Obtain the simplest form of Models 9 and 10 (note that, for convenience, we consider three types of confidence levels $\theta_{m_{i}}, \theta_{n_{j}}, \theta_{t_{K}}$ for constraints which belong to $\theta_{r}$ ).

(2) Solve the crisp MFCTPs (Models 9 and 10) by using fuzzy programming described in Section 6 .

(3) Obtain the optimal solution of each objective function (using, e.g., LINGO, as in our case).

\subsection{Application of proposed MFFTP}

To display the effectiveness of our designed fuzzy MFFTP (Model 3), we consider a real-life fuzzy bi-objective version of the MFFTP (i.e., a fuzzy BFFTP). When we set $K=1,2$, in Model 3, then our fuzzy MFFTP is reduced to a fuzzy BFFTP. The parameters of the fuzzy BFFTP (variable transportation cost, fixed-charge, investment amount, damaged rate, transportation time, supply, demand) are taken as fuzzy in nature. The objective functions $\tilde{Z}_{1}$ and $\tilde{Z}_{2}$ are described in the following manner:

$\tilde{Z}_{1}$ : minimize the ratio of $\{$ total transporting cost/investment $\}$;

$\tilde{Z}_{2}$ : minimize the ratio of $\{$ damaged rate of goods/time $\}$.

The fuzzy BFFTP can, therefore, is depicted as:

$$
\begin{aligned}
& \tilde{z}_{1}\left(x_{i j}, \eta\left(x_{i j}\right)\right)=\sum_{i=1}^{m} \sum_{j=1}^{n}\left(\tilde{c}_{i j} x_{i j}+\tilde{f}_{i j} \eta\left(x_{i j}\right)\right), \\
& \tilde{z}_{2}\left(x_{i j}\right)=\sum_{i=1}^{m} \sum_{j=1}^{n} \tilde{d}_{i j} x_{i j}, \\
& \tilde{g}_{1}\left(x_{i j}\right)=\sum_{i=1}^{m} \sum_{j=1}^{n} \tilde{p}_{i j} x_{i j}, \\
& \tilde{g}_{2}\left(x_{i j}\right)=\tilde{t}_{i j} \eta\left(x_{i j}\right) \\
& \eta\left(x_{i j}\right)=\left\{\begin{array}{l}
0 \text { if } x_{i j}=0, \\
1 \text { if } x_{i j}>0, \\
x_{i j} \geq 0, \quad i=1,2, \ldots, m ; j=1,2, \ldots, n,
\end{array}\right.
\end{aligned}
$$

with the same constraints of Models 5 or 9 or 10 , for $K=1,2$.

Here $\tilde{c}_{i j}, \tilde{f}_{i j}$ and $\tilde{p}_{i j}$ are the fuzzy versions of the variable and fixed transportation costs, and total investment whereas $\tilde{d}_{i j}$ and $\tilde{t}_{i j}$ denote the fuzzy deterioration rate and fuzzy transportation time independent of amount transported) to transport a unit commodity from source $i$ to destination $j$, respectively.

Remark 6.3. In the proposed model, the supplier/manufacturer of the company is the DM who pursues to optimize the model. It is also mentioned that supplier/manufacturer of the company will gain maximum benefit from the designed model.

\section{Application example: a practical transportation problem}

\subsection{Description of the problem}

A reputed Apple supplier company in India collects a large amount (around 7.58 lac metric tons) of apples throughout the year from Srinagar in Jammu and Kashmir, Shimla in Himachal Pradesh, Dehradun in Uttarakhand. To produce apple products for production company and to fulfill daily demand of markets that amounts 
of collecting apples are supplied to four demand points namely, Kolkata in West Bengal, Mumbai in Maharashtra, Gandhinagar in Gujarat, Hyderabad in Telangana. The supplier company transports apples from collection centers to demand points by large tracks/medium tracks through highways. For these cases, supplier company would recompense a definite amount of toll charge to Indian Highway Authority (i.e., fixed-charge is considered in transporting system). The DM desires to minimize (i) the total transporting cost concerning to total investment, and (ii) the damaged rate of goods with respect to transportation time (from origins to destinations). The transportation cost and investment in US\$, fixed-charge in US\$ for an unlock path, damaged rate in kilogram ( 1 damaged unit $=5$ kilograms), transportation time in hour are considered. The DM is also interested to find the amount of apples in ton to be transported from the $i$ th collecting center to the $j$ th demand point so as to satisfy the total requirement. The data are shown in Tables 3-5 to describe the whole problem in fuzzy sense.

\subsection{Discussion of model results}

This subsection discusses the optimal solutions of the identical crisp Models 5, 9 and 10 for $K=1$, 2, which are also the optimal solutions of Model 4 and as well as Model 3 for $K=1,2$.

(i) Using the data from Tables 3-5 in the formulated Model 5 for $K=1,2$; utilizing the methodology presented in Section 6.1, we obtain the results which are shown in Table 6.

(ii) Using the data from Tables 3-5 in the prescribed Models 9 and 10 for $K=1,2$, and utilizing the methodology presented in Section 6.2. To solve these models, we set confidence levels of $\delta_{1}=\delta_{2}=0.85$ and confidence levels of $\theta_{m_{1}}=\theta_{m_{2}}=\theta_{m_{3}}=0.90, \theta_{n_{1}}=\theta_{n_{2}}=\theta_{n_{3}}=0.90$ and $\theta_{t_{1}}=\theta_{t_{2}}=0.90$ (choice of the $\mathrm{DM}$, one can choice different values for confidence levels). So, we obtain the simplest form of Models 9 and 10 and the obtained results which are shown in Table 6 .

We can infer from Table 6 that the optimal solutions to the proposed MFFTP (i.e., Model 3 ) for $K=1,2$ is $(0.45,0.20)$, which is more effective than $(0.52,0.29)$ and $(0.54,0.49)$ as these are minimum value. Moreover, our suggested FCRA technique (in the case of LLA-MFCTP model) gives better optimal solution than existing method (i.e., Arya et al. [3] approach). We also observe that the optimal solution of proposed MFFTP derived from using FCRA is better than the optimal solution given by the RR technique, which is graphically shown in Figure 3. Here, we expand the feasible domain (for an optimal solution) of the proposed MFFTP which lies on LA, i.e., $(0.45,0.20)$; and on the UA, i.e., $(0.52,0.29)$. But a better optimal solution has occurred when the case of LLA-MFCTP (i.e., Model 9) for $K=1,2$ is considered.

The primary object of the DM of a supplier company is to supply the product timely in which the transportation cost and damaged rate of goods are minimized with respect to the investment and delivery time respectively. Here, the objective functions $\left(Z_{1}\right.$ and $\left.Z_{2}\right)$ in ratio form are minimized. To overcome the special situations in a transportation system, feasible solution space is extended and partitioned by using FCRA technique. In normal case the value of the objective functions are taken form Model 9 (i.e., from 0.45, 0.20) and for special case the value of the the objective function are chosen from Model 10 (i.e., from 0.52, 0.29). Thus, this model provides an important concept to tackle uncertainty in practical ratio optimization problem.

\section{MANAGERIAL INSIGHTS}

This section presents some managerial benefits of the suggested research study. This paper addresses the central decision for ratio optimization in a transportation system. The values of the objective functions are obtained from the designed model in ratio forms which are more acceptable for managerial decision.

Generally, the parameters connected to a practical distribution process are inexact in nature. Herein, triangular fuzzy numbers are utilized to tackle impreciseness of the parameters. Rough set approximation technique is adopted to extend and partition (in the sense of "surely" and "possible" cases) the region of optimal solution. These are the key strategies for a company's DM to tackle uncertainty in the real-world delivery system. "Surely" and "possible" solutions extracted from LA and UA models, respectively deal with the normal and 
TABLE 3. Triangular fuzzy values of shipping cost $\left(\tilde{c}_{i j}\right)$ and fixed-charge $\left(\tilde{f}_{i j}\right)$.

\begin{tabular}{ccccc}
\hline \hline & Destination-1 & Destination-2 & Destination-3 & Supply \\
\hline Origin-1 & $(3,4,5)$, & $(6,7,8)$, & $(5,7,9)$, & $(24,26,28)$ \\
& $(6,7,8)$ & $(8,9,10)$ & $(9,10,11)$ & \\
Origin-2 & $(8,9,10)$, & $(10,11,12)$, & $(6,8,10)$, & $(30,32,34)$ \\
& $(10,11,12)$ & $(12,13,14)$ & $(11,12,13)$ & \\
Origin-3 & $(6,10,14)$, & $(11,12,13)$, & $(10,12,14)$, & $(32,34,36)$ \\
& $(9,12,15)$ & $(13,14,15)$ & $(13,15,17)$ & \\
Demand & $(18,20,22)$ & $(25,26,27)$ & $(31,32,33)$ & \\
\hline
\end{tabular}

TABLE 4. Triangular fuzzy values of investment amount $\left(\tilde{p}_{i j}\right)$.

\begin{tabular}{ccccc}
\hline \hline & Destination-1 & Destination-2 & Destination-3 & Supply \\
\hline Origin-1 & $(8,9,10)$ & $(14,15,16)$ & $(12,13,14)$ & $(24,26,28)$ \\
Origin-2 & $(16,18,20)$ & $(18,19,20)$ & $(15,17,19)$ & $(30,32,34)$ \\
Origin-3 & $(20,21,22)$ & $(20,22,24)$ & $(17,18,19)$ & $(32,34,36$ \\
Demand & $(18,20,22)$ & $(25,26,27)$ & $(31,32,33)$ & \\
\hline
\end{tabular}

TABLE 5. Triangular fuzzy values of damaged rate $\left(\tilde{d}_{i j}\right)$ and time of transportation $\left(\tilde{t}_{i j}\right)$.

\begin{tabular}{ccccc}
\hline \hline & Destination-1 & Destination-2 & Destination-3 & Supply \\
\hline Origin-1 & $(1,2,3)$, & $(3,4,5)$, & $(2,3,4)$, & $(24,26,28)$ \\
& $(12,13,14)$ & $(14,15,16)$ & $(17,18,19)$ & \\
Origin-2 & $(4,5,6)$, & $(7,8,9)$, & $(6,7,8)$, & $(30,32,34)$ \\
& $(13,15,17)$ & $(16,18,20)$ & $(17,19,21)$ & \\
Origin-3 & $(5,7,9)$ & $(2,4,6)$, & $(5,6,7)$, & $(32,34,36)$ \\
& $(19,20,21)$ & $(20,21,22)$ & $(18,20,22)$ & \\
Demand & $(18,20,22)$ & $(25,26,27)$ & $(31,32,33)$ & \\
\hline
\end{tabular}

TABLE 6. Pareto-optimal solutions of the proposed MFFTP for $K=1,2$.

\begin{tabular}{lcc}
\hline \hline Technique (Applied to) & Optimal values of the $Z_{1}, Z_{2}$ & Values of $\lambda$ and $s$ \\
\hline Arya et al. [3] approach & $0.63,0.23$ & $s=0.0006$ \\
RR (Model 5) & $0.54,0.49$ & $0.75,0.0007$ \\
FCRA (Model 9: LLA-MFCTP) & $0.45,0.20$ & $0.70,0.0008$ \\
FCRA (Model 10: LUA-MFCTP) & $0.52,0.29$ & $0.61,0.0007$ \\
\hline
\end{tabular}

special cases of the practical transportation system which is an important blueprint for a policy-making problem. It is suitable for a DM to choose preferable optimal solution in a ratio optimization problem.

\section{Conclusion and outlook}

In this paper, for the first time, the multiple objective fractional TP with additional cost function (i.e., MFFTP) has been designed by adopting two distinct forms of uncertainty and its domain of optimal solutions is expanded. This paper has been simultaneously optimized with two fractional objective functions of conflicting nature which are: total transportation cost/investment and damaged rate of product/time. Due to the presence 


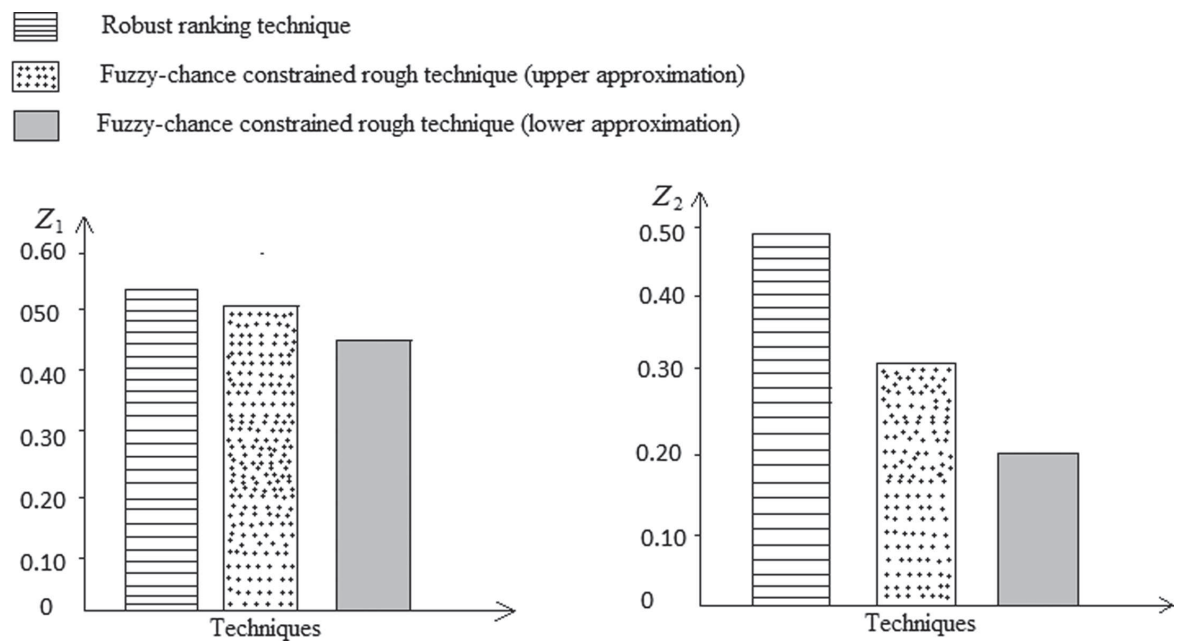

FiguRE 3 . Calculated values of $Z_{1}$ and $Z_{2}$ by techniques (represented by bars).

of inexactness of the data in the practical fractional FCTP, this article has been designed under two types of uncertain framework such as fuzzy and rough. Rough approximation technique has been used to tackle different situations like, "surely" and "possible" situations arise in real-world transportation system which make the policy-making system more flexible. Thus, it provides a better strategy for managerial decision and it is a cost-saving model.

The suggested fuzzy MFFTP has been converted into an identical crisp form by introducing two techniques, namely, RR and FCRA. FCRA has been used to divide the crisp form of the fuzzy MFCTP into two distinct forms, i.e., LLA-MFCTP and LUA-MFCTP. From the LAM and UAM of the MFCTP, it has been displayed that the feasible domain of the MFCTP (also, that of MFFTP) can be expanded as well as divided to provide more preferable optimal solutions. To show the effectiveness of our proposed MFFTP, we have compared the solutions between the solutions extracted from the RR and FCRA techniques, and also the existing method [3]. One of the limitations of the FCRA technique is that conversion procedure from fuzzy model to similar crisp model is slightly lengthy. Moreover, the proposed model is formulated under a single-stage system.

As an outlook, the proposed models can be extended to a multi-stage system [31] and various types of the transportation, fractional optimization as well as portfolio problems [15]. Besides, researchers can further analyze the multiple objectives fractional solid FCTP under intuitionistic fuzzy [12] or rough environments [30].

Acknowledgements. The authors are very thankful to the respected Editor-in-Chief Professor Nelson Maculan and the anonymous reviewers for their invaluable suggestions, which have improved the quality of the article significantly.

Conflict of interest. The authors stated that they have no difference of opinion to publish this article.

\section{REFERENCES}

[1] Y. Almogy and O. Levin, The fractional fixed charge problem. Nav. Res. Logist. Q. 18 (1971) 307-315.

[2] P. Anukokila, B. Radhakrishnan and A. Anju, Goal programming approach for solving multi-objective fractional transportation problem with fuzzy parameters. RAIRO-Oper. Res. 53 (2019) 157-178.

[3] R. Arya, P. Singh, S. Kumari and M.S. Obaidat, An approach for solving fully fuzzy multi-objective linear fractional optimization problems. Soft Comput. 24 (2020) 9105-9119.

[4] R.E. Bellman and L.A. Zadeh, Decision making in fuzzy environment. Manage. Sci. 17 (1970) 141-164.

[5] D. Bhati and P. Singh, Branch and bound computational method for multi-objective linear fractional optimization problem. Neural Comput. Appl. 28 (2017) 3341-3351. 
[6] A. Charnes and W.W. Cooper, Programming with linear fractional functionals. Nav. Res. Logist. Q. 9 (1962) $181-186$.

[7] M. Chakraborty and S. Gupta, Fuzzy mathematical programming for multi objective linear fractional programming problem. Fuzzy Set. Syst. 125 (2002) 335-342.

[8] C.-T. Chang, Fuzzy linearization strategy for multiple objective linear fractional programming with binary utility functions. Comput. Ind. Eng. 112 (2017)437-446.

[9] S.K. Das, S.A. Edalatpanah and T. Mandal, A proposed model for solving fuzzy linear fractional programming problem: Numerical point of view. J. Comput. Sci. 25 (2018) 367-375.

[10] D. Dubois, H. Prade, H. Farreny, R. Martin-Clouaire, C. Testemale and E. Harding, Possibility theory. Plenum Press, New York (1988).

[11] A. Ebrahimnejad, S.J. Ghomi and S.M. Mirhosseini-Alizamini, A revisit of numerical approach for solving linear fractional programming problem in a fuzzy environment. Appl. Math. Model. 57 (2018) 459-473.

[12] S. Ghosh, S.K. Roy, A. Ebrahimnejad and J.L. Verdegay, Multi-objective fully intuitionistic fuzzy fixed-charge solid transportation problem. Complex Intell. Syst. 7 (2021) 1009-1023. https://doi.org/10.1007/s40747-020-00251-3.

[13] S. Ghosh and S.K. Roy, Fuzzy-rough multi-objective product blending fixed-charge transportation problem with truck load constraints through transfer station. RAIRO-Oper. Res. 55 (2021) S2923-S2952.

[14] A. Goli, H.K. Zara, T.R. Moghaddam and A. Sadegheih, Multiobjective fuzzy mathematical model for a financially constrained closed-loop supply chain with labor employment. Comput. Intell. 36 (2020) 4-34.

[15] A. Goli, H.K. Zara, T.R. Moghaddam and A. Sadegheih, Hybrid artificial intelligence and robust optimization for a multiobjective product portfolio problem Case study: The dairy products industry. Comput. Ind. Eng. 137 (2019) 106090.

[16] A. Goli and B. Malmir, A covering tour approach for disaster relief locating and routing with fuzzy demand. Int. J. Intell. Transp. Syst. Res. 18 (2020) 140-152.

[17] A. Goli, E.B. Tirkolaee and N.S. Aydin, Fuzzy integrated cell formation and production scheduling considering automated guided vehicles and human factors. IEEE Trans. Fuzzy Syst. (2020). https://doi.org/10.1109/TFUZZ.2021.3053838.

[18] W.M. Hirsch and G.B. Dantzig, The fixed charge problem. Nav. Res. Logist. Q. 15 (1968) 413-424.

[19] H. Jiao and S. Liu A new linearization technique for minmax linear fractional programming. Int. J. Comput. Math. 91 (2014) 1730-1743.

[20] P. Kaur, V. Verma and K. Dahiya Capacitated two-stage time minimization transportation problem with restricted flow. RAIRO-Oper. Res. 51 (2017) 447-467.

[21] L. Li and K.K. Lai, A fuzzy approach to the multi-objective transportation problem. Comput. Oper. Res. 27 (2000), 43-57.

[22] B. Liu, Theory and practice of uncertain programming. Physica-Verlag, Heidelberg (2002).

[23] B. Liu and K. Iwamura, Chance constrained programming with fuzzy parameters. Fuzzy Set. Syst. 94 (1998) $227-237$.

[24] B. Liu and Y.K. Liu, Expected value of fuzzy variable and fuzzy expected value model. IEEE Trans. Fuzzy Syst. 10 (2002) $445-450$.

[25] A. Mahmoodirad, R. Dehghan and S. Niroomand, Modelling linear fractional transportation problem in belief degree based uncertain environment. J. Exp. Theor. Artif. Intell. 31 (2018) 1-16.

[26] G. Maity and S.K. Roy, Solving a multi-objective transportation problem with nonlinear cost and multi-choice demand. Int. J. Manag. Sci. Eng. Manag. 11 (2016) 62-70.

[27] G. Maity, D. Mardanya, S.K. Roy and G.W. Weber, A new approach for solving dual-hesitant fuzzy transportation problem with restrictions. Sadhana 44 (2019) 1-11. https://doi.org/10.1007/s12046-018-1045-1.

[28] S. Midya and S.K. Roy, Solving single-sink fixed-charge multi-objective multi-index stochastic transportation problem. Am. J. Math. Manag. Sci. 33 (2014) 300-314.

[29] S. Midya and S.K. Roy, Analysis of interval programming in different environments and its application to fixed-charge transportation problem. Discrete Math. Algorithm Appl. 9 (2017) 750040.

[30] S. Midya and S.K. Roy, Multi-objective fixed-charge transportation problem using rough programming. Int. J. Oper. Res. 37 (2020) 377-395.

[31] S. Midya, S.K. Roy and V.F. Yu, Intuitionistic fuzzy multi-stage multi-objective fixed-charge solid transportation problem in a green supply chain. Int. J. Mach. Learn. Cybern. 12 (2021) 699-717.

[32] S. Mishra, Weighting method for bi-level fractional programming problems. Eur. J. Oper. Res. 183 (2007) $296-302$.

[33] B. Mishra, K.A. Nishad and S.R. Singh, Fuzzy multi-fractional programming for land use planning in agricultural production system. Fuzzy Info. Eng. 6 (2014) 245-262.

[34] T. Paksoy, N.Y. Pehlivan, and E. Özceylan, Application of fuzzy optimization to a supply chain network design: A case study of an edible vegetable oils manufacturer. Appl. Math. Model. 36 (2012) 2762-2776.

[35] A. Paul, M. Pervin, S.K. Roy, G.W. Weber and A. Mirzazadeh, Effect of price-sensitive demand and default risk on optimal credit period and cycle time for a deteriorating inventory model. RAIRO-Oper. Res. 55 (2021) S2575-S2592.

[36] Z. Pawlak, Rough sets. Int. J. Info. Comput. Sci. 11 (1982) 341-356.

[37] S.K. Roy, G. Maity, G.W. Weber and S.Z.A. Gök, Conic scalarization approach to solve multi-choice multi-objective transportation problem with interval Goal. Ann. Oper. Res. 253 (2017) 599-620.

[38] S.K. Roy, S. Midya and V.F. Yu, Multi-objective fixed-charge transportation problem with random rough variables. Int. J. Uncertain. Fuzziness Knowledge-Based Syst. 26 (2018) 971-996.

[39] S.K. Roy and S. Midya, Multi-objective fixed-charge solid transportation problem with product blending under intuitionistic fuzzy environment. Appl. Intell. 49 (2019) 3524-3538. 
[40] S.K. Roy, S. Midya and G.W. Weber, Multi-objective multi-item fixed-charge solid transportation problem under twofold uncertainty. Neural Comput. Appl. 31 (2019) 8593-8613.

[41] S. Sagratella, M. Schmidt and Sudermann-Merx, N. The noncooperative fixed charge transportation problem. Eur. J. Oper. Res. 284 (2019) 373-382.

[42] S. Schaible, Fractional programming I: Duality. Manag. Sci. 22 (1976) 858-867.

[43] M. Sivri, I. Emiroglu, C. Guler and F. Tasci, A solution proposal to the transportation problem with the linear fractional objective function. In: Proc. of the 4th IEEE International Conference on Modeling, Simulation and Applied Optimization, Kuala Lumpur, Malaysia (2011).

[44] B. Stanojević and M. Stanojević, Comment on "Fuzzy mathematical programming for multi-objective linear fractional programming problem". Fuzzy Set. Syst. 246 (2014) 156-159.

[45] Z. Tao and J. Xu, A class of rough multiple objective programming and its application to solid transportation problem. Info. Sci. 188 (2012) 215-235.

[46] M.D. Toksari, Taylor series approach to fuzzy multi-objective linear fractional programming. Info. Sci. 178 (2008) $1189-1204$.

[47] M. Upmanyu and R.R. Saxena, On solving a multi-objective fixed charge problem with imprecise fractional objectives. Appl. Soft Comput. 40 (2016) 64-69.

[48] E.B. Tirkolaee, A. Goli, A. Faridnia, M. Soltani, and G.W. Weber, Multi-objective optimization for the reliable pollutionrouting problem with cross-dock selection using Pareto-based algorithms. J. Clean. Prod. 276 (2020) 122927.

[49] P. Vasant, G.W. Weber and V.N. Dieu. Handbook of Research on Modern Optimization Algorithms and Applications in Engineering and Economics. IGI Global, Hershey, PA (2016). https://doi.org/10.4018/978-1-4666-9644-0.

[50] C. Veeramani and M. Sumathi, Fuzzy mathematical programming approach for solving fuzzy linear fractional programming problem. RAIRO-Oper. Res. 48 (2014) 109-122.

[51] F. Xie and R. Jia, Nonlinear fixed charge transportation problem by minimum cost flow-based genetic algorithm. Comput. Ind. Eng. 63 (2012) 763-778.

[52] J. Xu and Z. Tao, Rough multiple objective decision making. Taylor and Francis Group, CRC Press, USA (2012).

[53] J. Xu and L. Zhao, A class of fuzzy rough expected value multi-objective decision making model and its application to inventory problems. Comput. Math. Appl. 56 (2008) 2107-2119.

[54] R.R. Yager, A procedure for ordering fuzzy subsets of the unit interval. Info. Sci. 24 (1981) $143-161$.

[55] L.A. Zadeh, Fuzzy sets. Info. Control 8 (1965), 338-353.

[56] L.A. Zadeh, Fuzzy sets as a basic for theory of possibility. Fuzzy Set. Syst. 1 (1978) 3-28.

[57] H.J. Zimmermann, Fuzzy programming and linear programming with several objective functions. Fuzzy Set. Syst. 1 (1978) $45-55$. 